\title{
On hyperbolic equations with double characteristics in the presence of transition
}

\author{
Annamaria Barbagallo* and Vincenzo Esposito
}

\section{"Correspondence:}

annamaria.barbagallo@unina.it Department of Mathematics and Applications "R. Caccioppoli",

University of Naples "Federico II", via Cintia - Monte S. Angelo, Naples, 80126 , Italy

\begin{abstract}
The paper deals with the study of the Cauchy problem for a class of hyperbolic second order operators with double characteristics in the presence of a transition. In particular, we obtain some a priori local estimates and, by means of these estimates, we prove local and global existence theorems.
\end{abstract}

MSC: Primary 35L20; secondary 35B45

Keywords: Sobolev spaces; Cauchy problem; hyperbolic equations; pseudodifferential operators

\section{Introduction}

Let $\Omega=\left[0,+\infty\left[\times \Omega_{0}, \Omega_{0}\right.\right.$ be an open subset of $\mathbb{R}^{n}(n \geq 2)$. Let $x=\left(x_{0}, x_{1}, \ldots, x_{m}, x_{m+1}, \ldots\right.$, $\left.x_{n}\right)=\left(x_{0}, x^{\prime}, x^{\prime \prime}\right) \in \Omega$, where we set $x^{\prime}=\left(x_{1}, \ldots, x_{m}\right) \in \Omega^{\prime}$ and $x^{\prime \prime}=\left(x_{m+1}, \ldots, x_{n}\right) \in \Omega^{\prime \prime}, \Omega^{\prime}$ is the projection of $\Omega_{0}$ on the hyperplane $x^{\prime \prime}=0$ and $\Omega^{\prime \prime}$ is the projection of $\Omega_{0}$ on the hyperplane $x^{\prime}=0$. Let us consider the following class of hyperbolic second order operators with double characteristics in the presence of a transition:

$$
P=D_{x_{0}}^{2}-\operatorname{Div}_{x^{\prime}}\left(A\left(x^{\prime}, x^{\prime \prime}\right) D_{x^{\prime}}\right)-\left(x_{0}+\lambda-\alpha\left(x^{\prime}\right)\right)^{2} \operatorname{Div}_{x^{\prime \prime}}\left(B\left(x^{\prime \prime}\right) D_{x^{\prime \prime}}\right)+\gamma(x), \quad \text { in } \Omega
$$

with $C^{\infty}$ coefficients, where $D_{x_{j}}=\frac{1}{i} \partial_{x_{j}}, j=0,1, \ldots, n, D_{x^{\prime}}=\frac{1}{i} \nabla_{x^{\prime}}=\left(D_{x_{1}}, \ldots, D_{x_{m}}\right), D_{x^{\prime \prime}}=$ $\frac{1}{i} \nabla_{x^{\prime \prime}}=\left(D_{x_{m+1}}, \ldots, D_{x_{n}}\right), \operatorname{Div}_{x^{\prime}}=\frac{1}{i} \operatorname{div}_{x^{\prime}}, \operatorname{Div}_{x^{\prime \prime}}=\frac{1}{i} \operatorname{div}_{x^{\prime \prime}}$ and $\lambda$ is a positive parameter.

For $\xi=\left(\xi_{0}, \xi_{1}, \ldots, \xi_{m}, \xi_{m+1}, \ldots, \xi_{n}\right)=\left(\xi_{0}, \xi^{\prime}, \xi^{\prime \prime}\right)$, where we set $\xi^{\prime}=\left(\xi_{1}, \ldots, \xi_{m}\right)$, $\xi^{\prime \prime}=$ $\left(\xi_{m+1}, \ldots, \xi_{n}\right)$ and have fixed $\lambda$, let us denote by

$$
\begin{aligned}
p(x, \xi)= & -\xi_{0}^{2}+\sum_{i, j=1}^{m} a_{i j}\left(x^{\prime}, x^{\prime \prime}\right) \xi_{i}^{\prime} \xi_{j}^{\prime}+\sum_{h=1}^{m} \partial_{x_{h}} a_{i j}\left(x^{\prime}, x^{\prime \prime}\right) \xi_{h}^{\prime} \\
& +\left(x_{0}+\lambda-\alpha\left(x^{\prime}\right)\right)^{2} \sum_{i, j=m+1}^{n} b_{j}\left(x^{\prime \prime}\right) \xi_{i}^{\prime \prime} \xi_{j}^{\prime \prime} \\
& +\left(x_{0}+\lambda-\alpha\left(x^{\prime}\right)\right)^{2} \sum_{h=m+1}^{n} \partial_{x_{h}} b_{j}\left(x^{\prime \prime}\right) \xi_{h}^{\prime \prime}+\gamma(x)
\end{aligned}
$$

(c) 2016 Barbagallo and Esposito. This article is distributed under the terms of the Creative Commons Attribution 4.0 International License (http://creativecommons.org/licenses/by/4.0/), which permits unrestricted use, distribution, and reproduction in any medium, provided you give appropriate credit to the original author(s) and the source, provide a link to the Creative Commons license, and indicate if changes were made. 
the symbol of $P$, by $\Sigma$ the characteristic set

$$
\Sigma=\left\{\rho=(x, \xi) \in T^{*} \Omega: p(\rho)=0, \nabla p(\rho)=0\right\}
$$

where $T^{*} \Omega=\Omega \times\left(\mathbb{R}^{n} \backslash\{0\}\right)$ is the cotangent bundle related to $\Omega$, and by $F_{p}$ the fundamental matrix of $P$ at $\rho$, namely

$$
F_{p}(\rho)=\frac{1}{2}\left(\begin{array}{cc}
p_{x \xi}^{\prime \prime}(\rho) & p_{\xi \xi}^{\prime \prime}(\rho) \\
-p_{x x}^{\prime \prime}(\rho) & -p_{\xi x}^{\prime \prime}(\rho) y
\end{array}\right), \quad \forall \rho \in \Sigma .
$$

The spectrum of $F(\rho)$, which we denote by $\operatorname{Spec}(F(\rho))$, has a remarkable importance for the study of the well-posedness of the Cauchy-Dirichlet problem for $P$.

Let us note that (see [1])

$$
z \in \operatorname{Spec}(F(\rho)) \quad \Leftrightarrow \quad-z, \bar{z} \in \operatorname{Spec}(F(\rho))
$$

It is well known that $F(\rho)$ has only pure imaginary eigenvalues with a possible exception of a pair of non-zero real eigenvalues $\pm \lambda$ (see $[1,2]$ ). If $F(\rho)$ has a pair of non-zero real eigenvalues, we say that $P$ is effectively hyperbolic at $\rho$. If $F(\rho)$ has only pure imaginary eigenvalues and, moreover, if in the Jordan normal form of $F(\rho)$ corresponding to the eigenvalue 0 , there are only Jordan blocks of dimension 2, i.e., $\operatorname{Ker} F(\rho)^{2} \cap \operatorname{Im} F(\rho)^{2}=\{0\}$, we say that $P$ is non-effectively hyperbolic of type 1 at $\rho$. Instead, if $F(\rho)$ has only pure imaginary eigenvalues and, moreover, if in the Jordan normal form of $F(\rho)$ corresponding to the eigenvalue 0 , there is only a Jordan block of dimension 4 and no block of dimension 3 , i.e., $\operatorname{Ker} F(\rho)^{2} \cap \operatorname{Im} F(\rho)^{2}$ is 2 -dimensional, we say that $P$ is non-effectively hyperbolic of type 2 at $\rho$. Besides let us set

$$
\begin{aligned}
& \Sigma_{+}=\{\rho \in \Sigma: P \text { is effectively hyperbolic at } \rho\}, \\
& \Sigma_{-}=\{\rho \in \Sigma: P \text { is non-effectively hyperbolic of type } 1 \text { at } \rho\}, \\
& \Sigma_{0}=\{\rho \in \Sigma: P \text { is non-effectively hyperbolic of type } 2 \text { at } \rho\} .
\end{aligned}
$$

It is easy to verify

$$
\Sigma=\Sigma_{-} \sqcup \Sigma_{0} \sqcup \Sigma_{+}
$$

Finally we say that we have a transition exactly when at least two among the above sets are nonempty.

The Cauchy problem for hyperbolic operators with double characteristics has been widely studied by many authors either in the case in which $F_{p}(\rho)$ has two real nonzero eigenvalues $\forall \rho \in \Sigma$ or in the case in which all the nonzero eigenvalues of $F_{p}(\rho)$ are purely imaginary numbers, $\forall \rho \in \Sigma$ (see for instance [1, 3-9]). Recently, another class of hyperbolic second order operators with double characteristics has been considered in [2]. For this class the $C^{\infty}$ well-posedness of the Cauchy problem is studied. Moreover, Carleman estimates are obtained for non-effectively hyperbolic operators. In [10], for a different class of hyperbolic second order operators some energy estimates are established and the $C^{\infty}$ 
well-posedness of the Cauchy problem for non-effectively hyperbolic operators is studied. We emphasize that in [2] and [10] the authors obtain a priori estimates when $\Sigma=\Sigma_{-} \sqcup \Sigma_{0}$. Instead we get $a$ priori estimates when $\Sigma=\Sigma_{-} \sqcup \Sigma_{0} \sqcup \Sigma_{+}$or $\Sigma=\Sigma_{-} \sqcup \Sigma_{0}$ or $\Sigma=\Sigma_{0} \sqcup \Sigma_{+}$ or $\Sigma=\Sigma_{-}$or $\Sigma=\Sigma_{+}$. In fact, in the class of operators (1), studied also in [11, 12] and [13], both in the case in which $F_{p}(\rho)$ has two distinct real eigenvalues and in the case in which all the eigenvalues are purely imaginary numbers can occur. Namely, on the variety characteristic a transition from a case to another one can be considered. More precisely, if $p(x, \xi)=\xi_{0}^{2}-\sum_{j=1}^{m} \xi_{j}^{2}+\left(x_{0}+\lambda-\alpha\left(x^{\prime}\right)\right)^{2} \sum_{j=m+1}^{n} \xi_{j}^{2}$, setting $\beta(x)=x_{0}+\lambda-\alpha\left(x^{\prime}\right)$, if $\left|\nabla_{x^{\prime}} \alpha\left(x^{\prime}\right)\right|<1$ and $\beta(x)=0 \quad\left(\xi_{0}=\xi_{1}=\cdots=\xi_{m}=0, \sum_{j=m+1}^{n} \xi_{j}^{2}=1\right)$, then $F_{p}(\rho)$ has two distinct nonzero real eigenvalues. As a consequence, $P$ is effectively hyperbolic. Instead if $\left|\nabla_{x^{\prime}} \alpha\left(x^{\prime}\right)\right|>1$ and $\beta(x)=0\left(\xi_{0}=\xi_{1}=\cdots=\xi_{m}=0, \sum_{j=m+1}^{n} \xi_{j}^{2}=1\right), F_{p}(\rho)$ has two nonzero imaginary eigenvalues, then $P$ is non-effectively hyperbolic. Therefore $\Sigma_{+}$is the set of points of $\Sigma$ for which $\left|\nabla_{x^{\prime}} \alpha(x)\right|<1, \Sigma_{-}$is the set of points of $\Sigma$ for which $\left|\nabla_{x^{\prime}} \alpha(x)\right|>1$ and $\Sigma_{0}$ is the set of points of $\Sigma$ in which $\left|\nabla_{x^{\prime}} \alpha(x)\right|=1$. Hence, even if we consider the particular class of operators (1), we have a transition from effectively hyperbolic to non-effectively hyperbolic.

In [11], an a priori estimate for solutions of a class of hyperbolic equations depending on a parameter $\left(-\partial_{x_{0}}^{2}+\partial_{x_{1}}^{2}+\left(x_{0}+\lambda-\alpha\left(x_{1}\right)\right)^{2} \partial_{x_{2}}^{2}\right) u=f$ related to a Cauchy-Dirichlet problem is proved. Then in [14] energy estimates and existence and uniqueness results are established. For the Cauchy problem related to the same class of hyperbolic operators, a global existence and uniqueness theorem is obtained in $[12,13]$ and energy estimates for solutions are established in [15]. In this paper, we study the general class of hyperbolic second order operators with double characteristics in the presence of a transition (1). Under suitable assumptions on the coefficients that allow the transition on the variety characteristic, we obtain, first of all, a priori local estimate near the boundary and, then, distant from it. Such estimates allow us to prove existence theorems for the following Cauchy problem in the set $\Omega$ :

$$
\left\{\begin{array}{l}
P u=f, \quad \text { in } \Omega, \\
u\left(0, x^{\prime}, x^{\prime \prime}\right)=0, \quad \partial_{x_{0}} u\left(0, x^{\prime}, x^{\prime \prime}\right)=0,
\end{array}\right.
$$

see Section 6.

Let us assume that:

(i) all the coefficients $a_{i j}\left(x^{\prime}, x^{\prime \prime}\right), i=1, \ldots, m$, and $b_{j}\left(x^{\prime \prime}\right), j=m+1, \ldots, n$ of the operator (1) belong to $C^{\infty}\left(\Omega_{0}\right) \cap L^{\infty}\left(\Omega_{0}\right)$ and $C_{0}^{\infty}\left(\Omega^{\prime \prime}\right) \cap L^{\infty}\left(\Omega^{\prime \prime}\right)$, respectively, for every $k>0$;

(ii) setting $g\left(x^{\prime}\right)=\frac{\alpha\left(x^{\prime}\right)}{\operatorname{div}_{x^{\prime}} \bar{\alpha}\left(x^{\prime}\right)}$, where $\bar{\alpha}\left(x^{\prime}\right)$ is a vector with $m$ components equal to $\alpha\left(x^{\prime}\right)$, and $h\left(x^{\prime}\right)=1-\operatorname{div}_{x^{\prime}} \bar{g}\left(x_{1}\right), g, h \in C^{\infty}, h\left(x^{\prime}\right) \in\left[h_{1}, h_{2}\right], \forall x^{\prime} \in \Omega^{\prime}$, with $0<h_{1}<h_{2}<4 ;$

(iii) there exists $\lambda>0$ such that $\left|g\left(x^{\prime}\right)\right| \leq \lambda, \forall x^{\prime} \in \Omega^{\prime}$;

(iv) setting $C\left(x^{\prime}, x^{\prime \prime}\right)=\operatorname{div}_{x^{\prime}} \bar{A}\left(x^{\prime}, x^{\prime \prime}\right) g\left(x^{\prime}\right)+2\left[A\left(x^{\prime}, x^{\prime \prime}\right) \operatorname{div}_{x^{\prime}} \bar{g}\left(x^{\prime}\right)-\Lambda\left(x^{\prime}, x^{\prime \prime}\right)\right]$, for every $\left(x^{\prime}, x^{\prime \prime}\right) \in \Omega_{0}$, where $\Lambda\left(x^{\prime}, x^{\prime \prime}\right)$ is a matrix with $m$ columns equal to $A\left(x^{\prime}, x^{\prime \prime}\right) \nabla_{x^{\prime}} g\left(x^{\prime}\right)$, the matrices $A$ and $B$ are positive definite and $C$ is positive semidefinite, namely

$$
\begin{aligned}
& \exists L_{1} \geq m: A\left(x^{\prime}, x^{\prime \prime}\right) \xi^{\prime} \xi^{\prime} \geq L_{1}\left\|\xi^{\prime}\right\|^{2}, \quad \forall \xi^{\prime} \in \mathbb{R}^{m}, \\
& \exists L_{2} \geq 0: B\left(x^{\prime \prime}\right) \xi^{\prime \prime} \xi^{\prime \prime} \geq L_{2}\left\|\xi^{\prime \prime}\right\|^{2}, \quad \forall \xi^{\prime \prime} \in \mathbb{R}^{n-m}, \\
& C\left(x^{\prime}, x^{\prime \prime}\right) \xi^{\prime} \xi^{\prime} \geq 0, \quad \forall \xi^{\prime} \in \mathbb{R}^{m} .
\end{aligned}
$$


It is worth remarking that in the study of hyperbolic operators considered in this note, the major difficulties in order to establish a priori estimates regard to the case in which the function $\beta(x, \lambda)=x_{0}+\lambda-\alpha\left(x^{\prime}\right)$ assumes positive and negative values in $\bar{\Omega}$. Let us observe that if $m=1$, setting $A\left(x^{\prime}, x^{\prime \prime}\right)=\left(a\left(x^{\prime}, x^{\prime \prime}\right)\right)$, as a result $C\left(x^{\prime}, x^{\prime \prime}\right)=\operatorname{div}_{x^{\prime}} a\left(x^{\prime}, x^{\prime \prime}\right) g\left(x^{\prime}\right)$. Moreover, if $a\left(x^{\prime}, x^{\prime \prime}\right)$ is a constant function, then $C\left(x^{\prime}, x^{\prime \prime}\right)=0$. Therefore, if $m=1$ and $A\left(x^{\prime}, x^{\prime \prime}\right)$ is a constant function, assumption (iv) naturally occurs.

Example 1.1 Let $\alpha\left(x_{1}\right)=e^{\frac{x_{1}^{3}}{3}+x_{1}}$ be a function defined in $\mathbb{R}$ and let $P=D_{x_{0}}^{2}-D_{x_{1}}^{2}-\left(x_{0}+\right.$ $\left.\lambda-\alpha\left(x_{1}\right)\right)^{2} D_{x_{2}}^{2}$. It is easy to verify that $g\left(x_{1}\right)=\frac{1}{x_{1}^{2}+1}$ and $h\left(x_{1}\right)=\frac{2 x_{1}}{\left(x_{1}^{2}+1\right)^{2}}+1$ in $\mathbb{R}$. Let us remark that $1-\frac{3 \sqrt{3}}{8} \leq h\left(x_{1}\right) \leq 1+\frac{3 \sqrt{3}}{8}, \forall x_{1} \in \mathbb{R}$. Moreover, the assumption (iii) is satisfied if we choose $\lambda \geq 1$. Therefore, we have $\Sigma=\Sigma_{-} \sqcup \Sigma_{0} \sqcup \Sigma_{+}$, namely we have a transition from effectively hyperbolic to non-effectively hyperbolic.

Example 1.2 Let us consider the function $\alpha\left(x_{1}, x_{2}\right)=e^{a x_{1}+b x_{2}}$ in $\mathbb{R}^{2}$, where $a+b \neq 0$, and the operator

$$
P=D_{x_{0}}^{2}-\left(3 D_{x_{1}}^{2}+D_{x_{1} x_{2}}^{2}+4 D_{x_{2}}^{2}\right)-\left(x_{0}+\lambda-\alpha\left(x_{1}, x_{2}\right)\right)^{2} D_{x_{3}}^{2}-\left(x_{0}+\lambda-\alpha\left(x_{1}, x_{2}\right)\right)^{2} D_{x_{4}}^{2}
$$

in $\left[0,+\infty\left[\times \mathbb{R}^{4}\right.\right.$. We observe that $g\left(x_{1}, x_{2}\right)=\frac{1}{a+b}$ and $h\left(x_{1}, x_{2}\right)=1$ in $\mathbb{R}^{2}$. Let us remark that

$$
A=\left(\begin{array}{cc}
3 & \frac{1}{2} \\
\frac{1}{2} & 4
\end{array}\right), \quad B=\left(\begin{array}{ll}
1 & 0 \\
0 & 1
\end{array}\right), \quad C=\left(\begin{array}{ll}
0 & 0 \\
0 & 0
\end{array}\right) .
$$

As a consequence, $A+C=A$. The matrices $A$ and $B$ are defined positive with constants $L_{1}=\frac{5}{2}$ and $L_{2}=1$, respectively. Moreover, assumption (iii) holds if $\lambda \geq \frac{1}{|a+b|}$. Therefore, $\Sigma=\Sigma_{-} \sqcup \Sigma_{0} \sqcup \Sigma_{+}$, then we have transition.

Example 1.3 Let us consider the function $\alpha\left(x_{1}, x_{2}, x_{3}\right)=\left(x_{1}+x_{2}+x_{3}\right)^{2}$ in $]-k, k{ }^{3}$, with $k>0$ and the operator

$$
\begin{aligned}
P= & D_{x_{0}}^{2}-\left(4 D_{x_{1}}^{2}+4 D_{x_{2}}^{2}+4 D_{x_{3}}^{2}+\frac{1}{2} D_{x_{1} x_{2}}^{2}+\frac{1}{2} D_{x_{1} x_{3}}^{2}+\frac{1}{2} D_{x_{2} x_{3}}^{2}\right) \\
& -\left(x_{0}+\lambda-\alpha\left(x_{1}, x_{2}, x_{3}\right)\right)^{2} D_{x_{4}}^{2}
\end{aligned}
$$

in $\left[0,+\infty[\times]-k, k\left[{ }^{3} \times \mathbb{R}\right.\right.$. It is easy to verify that $g\left(x_{1}, x_{2}, x_{3}\right)=\frac{1}{6}\left(x_{1}+x_{2}+x_{3}\right)$ and $h\left(x_{1}, x_{2}\right.$, $\left.x_{3}\right)=\frac{1}{2}$ in $]-k, k\left[{ }^{3}\right.$. Moreover, as a result

$$
A=\left(\begin{array}{ccc}
4 & \frac{1}{4} & \frac{1}{4} \\
\frac{1}{4} & 4 & \frac{1}{4} \\
\frac{1}{4} & \frac{1}{4} & 4
\end{array}\right), \quad B=(1), \quad C=\left(\begin{array}{ccc}
\frac{5}{2} & -\frac{5}{4} & -\frac{5}{4} \\
-\frac{5}{4} & \frac{5}{2} & -\frac{5}{4} \\
-\frac{5}{4} & -\frac{5}{4} & \frac{5}{2}
\end{array}\right)
$$

The matrices $A$ and $B$ are positive definite with constants $L_{1}=\frac{7}{2}$ and $L_{2}=1$, respectively, and $C$ is positive semidefinite. Finally, assumption (iii) is ensured when $\lambda \geq \frac{1}{2} k$. For $k$ large enough, $\Sigma=\Sigma_{-} \sqcup \Sigma_{0} \sqcup \Sigma_{+}$results, namely we have transition from effectively hyperbolic to non-effectively hyperbolic. 
The paper is organized as follows. In Section 2 some preliminary notations are given. In Section 3 a priori estimates are proved. In Section 4, estimates in Sobolev spaces with $s<0$ by means of the pseudodifferential operator theory are obtained. Section 5 deals with a local existence theorem near the boundary. Then a regularity result for the solution $u$ to the Cauchy problem (2) is shown. At last a global existence result is proved in Section 6.

\section{Notations and preliminaries}

Let $\alpha=\left(\alpha_{0}, \alpha^{\prime}, \alpha^{\prime \prime}\right) \in \mathbb{N}_{0}^{n+1}$. We denote by $\partial^{\alpha}$ the derivative of order $|\alpha|$, while $\partial_{x_{j}}^{h}$ means, as usually, the derivative of order $h$ with respect to $x_{j}$ and $\partial_{x_{j}, x_{p}}^{h}$ denotes the derivative of order $h$ with respect to $x_{j}$ and $x_{p}$.

Let us denote by $(\cdot, \cdot),\|\cdot\|,\|\cdot\|_{H^{r}}\left(r \in \mathbb{N}_{0}\right)$ the $L^{2}$-scalar product, the $L^{2}$-norm and the $H^{r}$-norm, respectively.

$C_{0}^{\infty}(\bar{\Omega})$ is the space of the restrictions to $\bar{\Omega}$ of functions $\varphi$ belonging to $C_{0}^{\infty}\left(\mathbb{R}^{n+1}\right)$ such that $\varphi$ vanishes with all the derivatives in $\left[0,+\infty\left[\times \partial \Omega_{0}\right.\right.$.

Let $s \in \mathbb{R}$, let us denote by $\|\cdot\|_{H^{0,0, s}}$ the norm given by

$$
\begin{aligned}
& \|u\|_{H^{0,0, s}(\bar{\Omega})}^{2}=\frac{1}{(2 \pi)^{n-m}} \int_{0}^{+\infty} d x_{0} \int_{\mathbb{R}^{m}} d x^{\prime} \int_{\mathbb{R}^{n-m}}\left(1+\left|\xi^{\prime \prime}\right|^{2}\right)^{s}\left|\widehat{u}\left(x_{0}, x^{\prime}, \xi^{\prime \prime}\right)\right|^{2} d \xi^{\prime \prime}, \\
& \forall u \in C_{0}^{\infty}(\bar{\Omega}),
\end{aligned}
$$

where the Fourier transform is done only with respect to the variable $x_{2}$. Moreover, let us denote by $A_{s}$ the pseudodifferential operator, given by

$$
A_{s} u=\frac{1}{(2 \pi)^{n-m}} \int_{\mathbb{R}^{n-m}} e^{i x^{\prime \prime} \cdot \xi^{\prime \prime}}\left(1+\left|\xi^{\prime \prime}\right|^{2}\right)^{\frac{s}{2}} \widehat{u}\left(x_{0}, x_{1}, \xi^{\prime \prime}\right) d \xi^{\prime \prime}, \quad \forall u \in C_{0}^{\infty}(\bar{\Omega}) .
$$

Let us recall that $A_{s}: C_{0}^{\infty}(\bar{\Omega}) \rightarrow C^{\infty}(\bar{\Omega})$. For every $\varphi\left(x^{\prime \prime}\right) \in C_{0}^{\infty}\left(\mathbb{R}^{n-m}\right)$, the operator $\varphi A_{s} u$ extends as a linear continuous operator from $H_{\text {comp. }}^{0,0, r}(\bar{\Omega})$ to $H_{\text {loc }}^{0,0, r-s}(\bar{\Omega})$, where $r, s \in \mathbb{R}$ (see [16]). Moreover, denoted by $\mathcal{U}_{x^{\prime \prime}}$ the projection of $\operatorname{supp} u$ on the hyperplane $x^{\prime \prime}=0$, if $\operatorname{supp} u \subseteq \mathbb{R}^{m-n} \backslash \mathcal{U}_{x^{\prime \prime}}$, then $\varphi A_{s} u$ is regularizing with respect to the variable $x^{\prime \prime}$, namely as a result

$$
\begin{aligned}
& \left\|\varphi A_{s} u\right\|_{H^{0,0, r}} \leq c\|u\|_{H^{0,0, r^{\prime}}}, \\
& \quad \forall r, r^{\prime} \in \mathbb{R}, u \in C^{\infty}(\bar{\Omega}): \operatorname{supp} u \subseteq\left[0,+\infty\left[\times \Omega^{\prime} \times \Omega^{\prime \prime} \backslash \operatorname{supp} \varphi .\right.\right.
\end{aligned}
$$

Let us remark that the norms $\|u\|_{H^{0,0, s(\Omega)}}$ and $\left\|A_{s} u\right\|_{L^{2}(\Omega)}$ are equivalent.

Finally, let $s, p \in \mathbb{R}$, let us denote by $\|\cdot\|_{H^{s, p}}$ the norm given by

$$
\begin{aligned}
& \|u\|_{H^{p, s}(\bar{\Omega})}^{2}=\sum_{|h| \leq p} \int_{0}^{+\infty} d x_{0} \int_{\mathbb{R}^{m}} d x^{\prime} \int_{\mathbb{R}^{n-m}} \frac{1}{(2 \pi)^{n-m}}\left(1+\left|\xi^{\prime \prime}\right|^{2}\right)^{p}\left|\partial_{x_{0}, x^{\prime}}^{h} \widehat{u}\left(x_{0}, x^{\prime}, \xi^{\prime \prime}\right)\right|^{2} d \xi^{\prime \prime} \\
& \forall u \in C_{0}^{\infty}(\bar{\Omega}) .
\end{aligned}
$$

\section{A priori estimates}

Lemma 3.1 Let $\Omega_{k}=\left[0, k\left[\times \Omega_{0}\right.\right.$, for every $k>0$, let $u \in C_{0}^{\infty}\left(\bar{\Omega}_{k}\right)$, as a result

$$
\|u\|_{L^{2}\left(\Omega_{k}\right)} \leq 2 k\left\|\partial_{x_{0}} u\right\|
$$




$$
\begin{aligned}
& \left\|u\left(0, x^{\prime}, x^{\prime \prime}\right)\right\|_{L^{2}\left(\Omega_{0}\right)}^{2} \leq 4 k\left\|\partial_{x_{0}} u\right\|^{2} \\
& \|u\|_{L^{2}\left(\Omega_{k}\right)}^{2}+\left\|u\left(0, x^{\prime}, x^{\prime \prime}\right)\right\|_{L^{2}\left(\Omega_{0}\right)}^{2} \leq 4\left(k^{2}+k\right)\left\|\partial_{x_{0}} u\right\|_{L^{2}\left(\Omega_{k}\right)}^{2}
\end{aligned}
$$

Proof Let $u \in C_{0}^{\infty}(\bar{\Omega})$. We have

$$
\begin{aligned}
0 & =\int_{\Omega} \partial_{x_{0}}\left(x_{0} u^{2}(x)\right) d x \\
& =\int_{\Omega} u^{2}(x) d x+2 \int_{\Omega} x_{0} u(x) \partial_{x_{0}} u(x) d x
\end{aligned}
$$

and therefore

$$
\int_{\Omega} u^{2}(x) d x=-2 \int_{\Omega} x_{0} u(x) \partial_{x_{0}} u(x) d x
$$

In particular, in $\Omega_{k}$ we obtain

$$
\|u\|^{2} \leq 2 k\|u\|\left\|\partial_{x_{0}} u\right\|
$$

which implies (4). Analogously, by using the following equality:

$$
\int_{\Omega} \partial_{x_{0}} u^{2}(x) d x=-\int_{\Omega_{0}} u^{2}\left(0, x^{\prime}, x^{\prime \prime}\right) d x^{\prime} d x^{\prime \prime}
$$

we obtain (5). Finally, collecting (4) and (5), we have (6).

Now, we are able to prove the following a priori estimate.

Theorem 3.1 Let $\Omega_{k}=\left[0, k\left[\times \Omega_{0}\right.\right.$ be a subset of $\Omega$, where $k>0$. Let us suppose that $g, h$ satisfy (i), (ii), and (iii). Then there exists a constant c $>0$ such that

$$
\begin{aligned}
& \left\|\partial_{x_{0}} u\right\|+\sum_{j=1}^{m}\left\|\partial_{x_{j}} u\right\|+\sum_{j=m+1}^{n}\left\|\left(x_{0}+\lambda-\alpha\left(x^{\prime}\right)\right) \partial_{x_{j}} u\right\| \leq c(\|P u\|+\|u\|), \\
& \forall u \in C_{0}^{\infty}\left(\bar{\Omega}_{k}\right) .
\end{aligned}
$$

Proof Let us integrate by parts in the inner product

$$
\begin{aligned}
2\left(\left(x_{0}+\lambda\right) \partial_{x_{0}} u(x), P u\right)= & \left\|\partial_{x_{0}} u\right\|^{2}+\lambda \int_{\Omega_{0}}\left(\partial_{x_{0}} u\left(0, x^{\prime}, x^{\prime \prime}\right)\right)^{2} d x^{\prime} d x^{\prime \prime} \\
& -2 \int_{\Omega} A\left(x^{\prime}, x^{\prime \prime}\right) \nabla_{x^{\prime}} u(x) \cdot\left(x_{0}+\lambda\right) \nabla_{x^{\prime}} \partial_{x_{0}} u(x) d x \\
& -2 \int_{\Omega}\left(x_{0}+\lambda-\alpha\left(x^{\prime}\right)\right)^{2} B\left(x^{\prime \prime}\right) \nabla_{x^{\prime \prime}} u(x) \cdot\left(x_{0}+\lambda\right) \nabla_{x^{\prime \prime}} \partial_{x_{0}} u(x) d x \\
& -\int_{\Omega} \gamma(x) u^{2}(x) d x \\
& -\int_{\Omega} \partial_{x_{0}} \gamma(x)\left(x_{0}+\lambda\right) u^{2}(x) d x
\end{aligned}
$$




$$
\begin{aligned}
& \quad-\lambda \int_{\Omega_{0}} \gamma\left(0, x^{\prime}, x^{\prime \prime}\right) u^{2}\left(0, x^{\prime}, x^{\prime \prime}\right) d x^{\prime} d x^{\prime \prime} \\
& =\left\|\partial_{x_{0}} u\right\|^{2}+\lambda \int_{\Omega_{0}}\left(\partial_{x_{0}} u\left(0, x^{\prime}, x^{\prime \prime}\right)\right)^{2} d x^{\prime} d x^{\prime \prime} \\
& +\int_{\Omega} A\left(x^{\prime}, x^{\prime \prime}\right) \nabla_{x^{\prime}} u(x) \cdot \nabla_{x^{\prime}} u(x) d x \\
& +\lambda \int_{\Omega_{0}} A\left(x^{\prime}, x^{\prime \prime}\right) \nabla_{x^{\prime}} u\left(0, x^{\prime}, x^{\prime \prime}\right) \cdot \nabla_{x^{\prime}} u\left(0, x^{\prime}, x^{\prime \prime}\right) d x^{\prime} d x^{\prime \prime} \\
& +2 \int_{\Omega}\left(x_{0}+\lambda-\alpha\left(x^{\prime}\right)\right) B\left(x^{\prime \prime}\right) \nabla_{x^{\prime \prime}} u(x) \cdot\left(x_{0}+\lambda\right) \nabla_{x^{\prime \prime}} u(x) d x \\
& +\int_{\Omega}\left(x_{0}+\lambda-\alpha\left(x^{\prime}\right)\right)^{2} B\left(x^{\prime \prime}\right) \nabla_{x^{\prime \prime}} u(x) \cdot \nabla_{x^{\prime \prime}} u(x) d x \\
& +\lambda \int_{\Omega_{0}}\left(\lambda-\alpha\left(x^{\prime}\right)\right)^{2} B\left(x^{\prime \prime}\right) \nabla_{x^{\prime \prime}} u\left(0, x^{\prime}, x^{\prime \prime}\right) \\
& +\nabla_{x^{\prime \prime}} u\left(0, x^{\prime}, x^{\prime \prime}\right) d x^{\prime} d x^{\prime \prime} \\
& -\int_{\Omega} \gamma(x) u^{2}(x) d x-\int_{\Omega}\left(x_{0}+\lambda\right) u^{2}(x) \partial_{x_{0}} \gamma(x) d x \\
& -\lambda \int_{\Omega_{0}} \gamma\left(0, x^{\prime}, x^{\prime \prime}\right) u^{2}\left(0, x^{\prime}, x^{\prime \prime}\right) d x^{\prime} d x^{\prime \prime} .
\end{aligned}
$$

On the other hand, by integrating by parts in the inner product, we obtain

$$
\begin{aligned}
2\left(g\left(x^{\prime}\right) \operatorname{div}_{x^{\prime}} \bar{u}(x), P u\right)= & -2\left(g\left(x^{\prime}\right) \operatorname{div}_{x^{\prime}} \bar{u}(x), \partial_{x_{0}}^{2} u(x)\right) \\
& +2\left(g\left(x^{\prime}\right) \operatorname{div}_{x^{\prime}} \bar{u}(x), \operatorname{div}_{x^{\prime}} A\left(x^{\prime}, x^{\prime \prime}\right) \nabla_{x^{\prime}} u(x)\right) \\
& +2\left(g\left(x^{\prime}\right) \operatorname{div}_{x^{\prime}} \bar{u}(x),\left(x_{0}+\lambda-\alpha\left(x^{\prime}\right)\right)^{2} \operatorname{div}_{x^{\prime \prime}} B\left(x^{\prime \prime}\right) \nabla_{x^{\prime \prime}} u(x)\right) \\
& +2\left(g\left(x^{\prime}\right) \operatorname{div}_{x^{\prime}} \bar{u}(x), \gamma(x) u(x)\right) .
\end{aligned}
$$

Let us compute separately every inner product:

$$
\begin{aligned}
-2\left(g\left(x^{\prime}\right) \operatorname{div}_{x^{\prime}} \bar{u}(x), \partial_{x_{0}}^{2} u(x)\right)= & 2\left(g\left(x^{\prime}\right) \operatorname{div}_{x^{\prime}} \partial_{x_{0}} \bar{u}(x), \partial_{x_{0}} u(x)\right) \\
& +2 \int_{\Omega_{0}} \partial_{x_{0}} u\left(0, x^{\prime}, x^{\prime \prime}\right) g\left(x^{\prime}\right) \operatorname{div}_{x^{\prime}} \bar{u}\left(0, x^{\prime}, x^{\prime \prime}\right) d x^{\prime} d x^{\prime \prime} \\
= & -\int_{\Omega} \operatorname{div}_{x^{\prime}} \bar{g}\left(x^{\prime}\right)\left(\partial_{x_{0}} u(x)\right)^{2} d x \\
& +2 \int_{\Omega_{0}} \partial_{x_{0}} u\left(0, x^{\prime}, x^{\prime \prime}\right) g\left(x^{\prime}\right) \operatorname{div}_{x^{\prime}} \bar{u}\left(0, x^{\prime}, x^{\prime \prime}\right) d x^{\prime} d x^{\prime \prime} .
\end{aligned}
$$

For the second one, we have

$$
\begin{aligned}
& 2\left(g\left(x^{\prime}\right) \operatorname{div}_{x^{\prime}} \bar{u}(x), \operatorname{div}_{x^{\prime}} A\left(x^{\prime}, x^{\prime \prime}\right) \nabla_{x^{\prime}} u(x)\right) \\
& =-2 \sum_{h=1}^{m} \int_{\Omega} A\left(x^{\prime}, x^{\prime \prime}\right) \nabla_{x^{\prime}} u(x) \cdot g\left(x^{\prime}\right) \nabla_{x^{\prime}} \partial_{x_{h}} u(x) d x \\
& \quad-2 \sum_{h=1}^{m} \int_{\Omega} A\left(x^{\prime}, x^{\prime \prime}\right) \nabla_{x^{\prime}} u(x) \cdot \partial_{x_{h}} u(x) \nabla_{x^{\prime}} g\left(x^{\prime}\right) d x .
\end{aligned}
$$


Moreover, as a result

$$
\begin{aligned}
& -2 \sum_{h=1}^{m} \int_{\Omega} A\left(x^{\prime}, x^{\prime \prime}\right) \nabla_{x^{\prime}} u(x) \cdot g\left(x^{\prime}\right) \nabla_{x^{\prime}} \partial_{x_{h}} u(x) d x \\
& =2 \sum_{h=1}^{m} \int_{\Omega} A\left(x^{\prime}, x^{\prime \prime}\right) \nabla_{x^{\prime}} \partial_{x_{h}} u(x) \cdot g\left(x^{\prime}\right) \nabla_{x^{\prime}} u(x) d x \\
& \quad+2 \sum_{h=1}^{m} \int_{\Omega} \partial_{x_{h}} A\left(x^{\prime}, x^{\prime \prime}\right) \nabla_{x^{\prime}} u(x) \cdot g\left(x^{\prime}\right) \nabla_{x^{\prime}} u(x) d x \\
& \quad+2 \sum_{h=1}^{m} \int_{\Omega} A\left(x^{\prime}, x^{\prime \prime}\right) \nabla_{x^{\prime}} u(x) \cdot \partial_{x_{h}} g\left(x^{\prime}\right) \nabla_{x^{\prime}} u(x) d x
\end{aligned}
$$

and that implies

$$
\begin{aligned}
-2 & \sum_{h=1}^{m} \int_{\Omega} A\left(x^{\prime}, x^{\prime \prime}\right) \nabla_{x^{\prime}} u \cdot g\left(x^{\prime}\right) \nabla_{x^{\prime}} \partial_{x_{h}} u(x) d x \\
= & \sum_{h=1}^{m} \int_{\Omega} \partial_{x_{h}} A\left(x^{\prime}, x^{\prime \prime}\right) \nabla_{x^{\prime}} u(x) \cdot g\left(x^{\prime}\right) \nabla_{x^{\prime}} u(x) d x \\
& +\sum_{h=1}^{m} \int_{\Omega} A\left(x^{\prime}, x^{\prime \prime}\right) \nabla_{x^{\prime}} u(x) \cdot \partial_{x_{h}} g\left(x^{\prime}\right) \nabla_{x^{\prime}} u(x) d x .
\end{aligned}
$$

Substituting (12) in (11), we obtain

$$
\begin{aligned}
& 2\left(g\left(x^{\prime}\right) \operatorname{div}_{x^{\prime}} \bar{u}(x), \operatorname{div}_{x^{\prime}}\left(A\left(x^{\prime}, x^{\prime \prime}\right) \nabla_{x^{\prime}} u(x)\right)\right) \\
& =\sum_{h=1}^{m} \int_{\Omega} \partial_{x_{h}} A\left(x^{\prime}, x^{\prime \prime}\right) \nabla_{x^{\prime}} u(x) \cdot g\left(x^{\prime}\right) \nabla_{x^{\prime}} u(x) d x \\
& \quad+\sum_{h=1}^{m} \int_{\Omega} A\left(x^{\prime}, x^{\prime \prime}\right) \nabla_{x^{\prime}} u(x) \cdot \partial_{x_{h}} g\left(x^{\prime}\right) \nabla_{x^{\prime}} u(x) d x \\
& \quad-2 \sum_{h=1}^{m} \int_{\Omega} A\left(x^{\prime}, x^{\prime \prime}\right) \nabla_{x^{\prime}} u(x) \cdot \partial_{x_{h}} u(x) \nabla_{x^{\prime}} g\left(x^{\prime}\right) d x .
\end{aligned}
$$

We compute

$$
\begin{aligned}
& \sum_{h=1}^{m} \partial_{x_{h}} A\left(x^{\prime}, x^{\prime \prime}\right) \nabla_{x^{\prime}} u(x) \cdot g\left(x^{\prime}\right) \nabla_{x^{\prime}} u(x)+\sum_{h=1}^{m} A\left(x^{\prime}, x^{\prime \prime}\right) \nabla_{x^{\prime}} u(x) \cdot \partial_{x_{h}} g\left(x^{\prime}\right) \nabla_{x^{\prime}} u(x) \\
& \quad=\operatorname{div}_{x^{\prime}} \bar{A}\left(x^{\prime}, x^{\prime \prime}\right) \nabla_{x^{\prime}} u(x) \cdot g\left(x^{\prime}\right) \nabla_{x^{\prime}} u(x)+A\left(x^{\prime}, x^{\prime \prime}\right) \nabla_{x^{\prime}} u(x) \cdot \operatorname{div}_{x^{\prime}} \bar{g}\left(x^{\prime}\right) \nabla_{x^{\prime}} u(x) \\
& \quad=\left(\operatorname{div}_{x^{\prime}} \bar{A}\left(x^{\prime}, x^{\prime \prime}\right) g\left(x^{\prime}\right)+A\left(x^{\prime}, x^{\prime \prime}\right) \operatorname{div}_{x^{\prime}} \bar{g}\left(x^{\prime}\right)\right) \nabla_{x^{\prime}} u(x) \cdot \nabla_{x^{\prime}} u(x) .
\end{aligned}
$$

Moreover, as a result

$$
\sum_{h=1}^{m} A\left(x^{\prime}, x^{\prime \prime}\right) \nabla_{x^{\prime}} u \cdot \partial_{x_{h}} u \nabla_{x^{\prime}} g\left(x^{\prime}\right)=\Lambda\left(x^{\prime}, x^{\prime \prime}\right) \nabla_{x^{\prime}} u(x) \cdot \nabla_{x^{\prime}} u(x)
$$


where $\Lambda\left(x^{\prime}, x^{\prime \prime}\right)$ is a matrix with $m$ columns equal to $A\left(x^{\prime}, x^{\prime \prime}\right) \nabla_{x^{\prime}} g\left(x^{\prime}\right)$. Taking into account (13), (14), and (15), we obtain

$$
\begin{aligned}
& 2\left(g\left(x^{\prime}\right) \operatorname{div}_{x^{\prime}} \bar{u}(x), \operatorname{div}_{x^{\prime}} A\left(x^{\prime}, x^{\prime \prime}\right) \nabla_{x^{\prime}} u(x)\right) \\
& =\int_{\Omega}\left[\operatorname{div}_{x^{\prime}} \bar{A}\left(x^{\prime}, x^{\prime \prime}\right) g\left(x^{\prime}\right)+2 A\left(x^{\prime}, x^{\prime \prime}\right) \operatorname{div}_{x^{\prime}} \bar{g}\left(x^{\prime}\right)-2 \Lambda\left(x^{\prime}, x^{\prime \prime}\right)\right] \nabla_{x^{\prime}} u(x) \cdot \nabla_{x^{\prime}} u(x) d x \\
& \quad-\int_{\Omega} A\left(x^{\prime}, x^{\prime \prime}\right) \operatorname{div}_{x^{\prime}} \bar{g}\left(x^{\prime}\right) \nabla_{x^{\prime}} u \cdot \nabla_{x^{\prime}} u d x \\
& =\int_{\Omega} C\left(x^{\prime}, x^{\prime \prime}\right) \nabla_{x^{\prime}} u(x) \cdot \nabla_{x^{\prime}} u(x) d x-\int_{\Omega} A\left(x^{\prime}, x^{\prime \prime}\right) \nabla_{x^{\prime}} u \cdot \nabla_{x^{\prime}} u d x \\
& \quad+\int_{\Omega} h\left(x^{\prime}\right) A\left(x^{\prime}, x^{\prime \prime}\right) \nabla_{x^{\prime}} u \cdot \nabla_{x^{\prime}} u d x,
\end{aligned}
$$

where we have set $C\left(x^{\prime}, x^{\prime \prime}\right)=\operatorname{div}_{x^{\prime}} \bar{A}\left(x^{\prime}, x^{\prime \prime}\right) g\left(x^{\prime}\right)+2\left[A\left(x^{\prime}, x^{\prime \prime}\right) \operatorname{div}_{x^{\prime}} \bar{g}\left(x^{\prime}\right)-\Lambda\left(x^{\prime}, x^{\prime \prime}\right)\right]$, for every $\left(x^{\prime}, x^{\prime \prime}\right) \in \Omega_{0}$.

Let us consider

$$
\begin{aligned}
& 2\left(g\left(x^{\prime}\right) \operatorname{div}_{x^{\prime}} \bar{u}(x),\left(x_{0}+\lambda-\alpha\left(x^{\prime}\right)\right)^{2} \operatorname{div}_{x^{\prime \prime}}\left(\bar{B}\left(x^{\prime \prime}\right) \nabla_{x^{\prime \prime}} u(x)\right)\right) \\
& =-2 \sum_{h=1}^{m} \int_{\Omega}\left(x_{0}+\lambda-\alpha\left(x^{\prime}\right)\right)^{2} B\left(x^{\prime \prime}\right) \nabla_{x^{\prime \prime}} u(x) \cdot g\left(x^{\prime}\right) \nabla_{x^{\prime \prime}} \partial_{x_{h}} u(x) d x \\
& =2 \sum_{h=1}^{m} \int_{\Omega}\left(x_{0}+\lambda-\alpha\left(x^{\prime}\right)\right)^{2} B\left(x^{\prime \prime}\right) \nabla_{x^{\prime \prime}} \partial_{x_{h}} u(x) \cdot g\left(x^{\prime}\right) \nabla_{x^{\prime \prime}} u(x) d x \\
& \quad-4 \sum_{h=1}^{m} \int_{\Omega}\left(x_{0}+\lambda-\alpha\left(x^{\prime}\right)\right) \partial_{x_{h}} \alpha\left(x^{\prime}\right) B\left(x^{\prime \prime}\right) \nabla_{x^{\prime \prime}} u(x) \cdot g\left(x^{\prime}\right) \nabla_{x^{\prime \prime}} u(x) d x \\
& \quad+2 \sum_{h=1}^{m} \int_{\Omega}\left(x_{0}+\lambda-\alpha\left(x^{\prime}\right)\right)^{2} B\left(x^{\prime \prime}\right) \nabla_{x^{\prime \prime}} u(x) \cdot \partial_{x_{h}} g\left(x^{\prime}\right) \nabla_{x^{\prime \prime}} u(x) d x,
\end{aligned}
$$

from which it follows that

$$
\begin{aligned}
-2 & \sum_{h=1}^{m} \int_{\Omega}\left(x_{0}+\lambda-\alpha\left(x^{\prime}\right)\right)^{2} B\left(x^{\prime \prime}\right) \nabla_{x^{\prime \prime}} u \cdot g\left(x^{\prime}\right) \nabla_{x^{\prime \prime}} \partial_{x_{h}} u d x \\
= & -2 \sum_{h=1}^{m} \int_{\Omega}\left(x_{0}+\lambda-\alpha\left(x^{\prime}\right)\right) \partial_{x_{h}} \alpha\left(x^{\prime}\right) B\left(x^{\prime \prime}\right) \nabla_{x^{\prime \prime}} u \cdot g\left(x^{\prime}\right) \nabla_{x^{\prime \prime}} \partial_{x_{h}} u d x \\
& +\sum_{h=1}^{m} \int_{\Omega}\left(x_{0}+\lambda-\alpha\left(x^{\prime}\right)\right)^{2} B\left(x^{\prime \prime}\right) \nabla_{x^{\prime \prime}} u(x) \cdot \partial_{x_{h}} g\left(x^{\prime}\right) \nabla_{x^{\prime \prime}} u(x) d x .
\end{aligned}
$$

Substituting (18) in (17) and using assumption (ii), we have

$$
\begin{aligned}
& -2\left(g\left(x^{\prime}\right) \operatorname{div}_{x^{\prime}} \bar{u}(x),\left(x_{0}+\lambda-\alpha\left(x^{\prime}\right)\right)^{2} \operatorname{div}_{x^{\prime \prime}} \bar{B}\left(x^{\prime \prime}\right) \nabla_{x^{\prime \prime}} u(x)\right) \\
& =-2 \sum_{h=1}^{m} \int_{\Omega}\left(x_{0}+\lambda-\alpha\left(x^{\prime}\right)\right) \partial_{x_{h}} \alpha\left(x^{\prime}\right) B\left(x^{\prime \prime}\right) \nabla_{x^{\prime \prime}} u(x) \cdot g(x) \nabla_{x^{\prime \prime}} u(x) d x
\end{aligned}
$$




$$
\begin{aligned}
& +\sum_{h=1}^{m} \int_{\Omega}\left(x_{0}+\lambda-\alpha\left(x^{\prime}\right)\right)^{2} B\left(x^{\prime \prime}\right) \nabla_{x^{\prime \prime}} u(x) \cdot \partial_{x_{h}} g\left(x^{\prime}\right) \nabla_{x^{\prime \prime}} u(x) d x \\
= & -2 \int_{\Omega}\left(x_{0}+\lambda-\alpha\left(x^{\prime}\right)\right) B\left(x^{\prime \prime}\right) \nabla_{x^{\prime \prime}} u(x) \cdot \alpha(x) \nabla_{x^{\prime \prime}} u(x) d x \\
& +\int_{\Omega}\left(x_{0}+\lambda-\alpha\left(x^{\prime}\right)\right)^{2} B\left(x^{\prime \prime}\right) \nabla_{x^{\prime \prime}} u(x) \cdot \operatorname{div}_{x^{\prime}} \bar{g}\left(x^{\prime}\right) \nabla_{x^{\prime \prime}} u(x) d x \\
= & -2 \int_{\Omega}\left(x_{0}+\lambda-\alpha\left(x^{\prime}\right)\right) B\left(x^{\prime \prime}\right) \nabla_{x^{\prime \prime}} u(x) \cdot \alpha(x) \nabla_{x^{\prime \prime}} u(x) d x \\
& +\int_{\Omega}\left(x_{0}+\lambda-\alpha\left(x^{\prime}\right)\right)^{2} B\left(x^{\prime \prime}\right) \nabla_{x^{\prime \prime}} u(x) \cdot \nabla_{x^{\prime \prime}} u(x) d x \\
& -\int_{\Omega}\left(x_{0}+\lambda-\alpha\left(x^{\prime}\right)\right)^{2} B\left(x^{\prime \prime}\right) \nabla_{x^{\prime \prime}} u(x) \cdot h\left(x^{\prime}\right) \nabla_{x^{\prime \prime}} u(x) d x .
\end{aligned}
$$

Finally, we have

$$
\begin{aligned}
2\left(g\left(x^{\prime}\right) \operatorname{div}_{x^{\prime}} \bar{u}(x), \gamma(x) u(x)\right)= & -\int_{\Omega} \operatorname{div}_{x^{\prime}} \bar{\gamma}(x) g\left(x^{\prime}\right) u^{2}(x) d x \\
& -\int_{\Omega} \gamma(x) \operatorname{div}_{x^{\prime}} \bar{g}\left(x^{\prime}\right) u^{2}(x) d x \\
= & -\int_{\Omega} \operatorname{div}_{x^{\prime}} \bar{\gamma}(x) g\left(x^{\prime}\right) u^{2}(x) d x \\
& -\int_{\Omega} \gamma(x) u^{2}(x) d x \\
& +\int_{\Omega} \gamma(x) h\left(x^{\prime}\right) u^{2}(x) d x
\end{aligned}
$$

By adding (10), (16), (19), and (20), we have

$$
\begin{aligned}
2\left(\left(x_{0}+\lambda\right) \partial_{x_{0}} u+g\left(x^{\prime}\right) \operatorname{div}_{x^{\prime \prime}} \bar{u}, P u\right) \\
=\left\|\partial_{x_{0}} u\right\|^{2}+\int_{\Omega}\left(4-h\left(x^{\prime}\right)\right)\left(x_{0}+\lambda-\alpha\left(x^{\prime}\right)\right)^{2} B\left(x^{\prime \prime}\right) \nabla_{x^{\prime \prime}} u(x) \cdot \nabla_{x^{\prime \prime}} u(x) d x \\
\quad+\int_{\Omega_{0}}\left\{\lambda\left[\left(\partial_{x_{0}} u\left(0, x^{\prime}, x^{\prime \prime}\right)\right)^{2}+A\left(x^{\prime}, x^{\prime \prime}\right) \nabla_{x^{\prime}} u\left(0, x^{\prime}, x^{\prime \prime}\right) \cdot \nabla_{x^{\prime}} u\left(0, x^{\prime}, x^{\prime \prime}\right)\right]\right. \\
\left.+2 \partial_{x_{0}} u\left(0, x^{\prime}, x^{\prime \prime}\right) g\left(x^{\prime}\right) \operatorname{div}_{x^{\prime}} \bar{u}\left(0, x^{\prime}, x^{\prime \prime}\right)\right\} d x^{\prime} d x^{\prime \prime} \\
+\int_{\Omega}\left(h\left(x^{\prime}\right) A\left(x^{\prime}, x^{\prime \prime}\right)+C\left(x^{\prime}, x^{\prime \prime}\right)\right) \nabla_{x^{\prime}} u \cdot \nabla_{x^{\prime}} u d x \\
+\int_{\Omega}\left(2 h\left(x^{\prime}\right)-3\right) \gamma(x) u^{2}(x) d x \\
\quad-\int_{\Omega}\left(x_{0}+\lambda\right) \partial_{x_{0}} \gamma(x) u^{2}(x) d x \\
\quad-\lambda \int_{\partial \Omega} \gamma\left(0, x^{\prime}, x^{\prime \prime}\right) u^{2}\left(0, x^{\prime}, x^{\prime \prime}\right) d x^{\prime} d x^{\prime \prime} \\
\quad-\int_{\Omega} \operatorname{div}_{x^{\prime}} \bar{g}\left(x^{\prime}\right) u^{2}(x) d x .
\end{aligned}
$$


Making use of assumptions (i), (ii), (iii), and (iv), we obtain

$$
\begin{aligned}
\left(\left(x_{0}+\lambda\right) \partial_{x_{0}} u+g\left(x^{\prime}\right) \operatorname{div}_{x^{\prime}} \bar{u}(x), P u\right) \\
\geq h_{1}\left\|\partial_{x_{0}} u\right\|^{2}+L_{1} h_{1} \sum_{j=1}^{m}\left\|\partial_{x_{j}} u\right\|^{2} \\
\quad+L_{2}\left(4-h_{2}\right) \sum_{j=1}^{n-m}\left\|\left(x_{0}+\lambda-\alpha\left(x^{\prime}\right)\right) \partial_{x_{j+m}} u\right\|^{2}+4\left(k^{2}+k\right) c\|u\|^{2}, \quad \forall u \in C_{0}^{\infty}\left(\bar{\Omega}_{k}\right),
\end{aligned}
$$

where $\Omega_{k}=\left[0, k\left[\times \Omega_{0}\right.\right.$, with $k>0$, from which (7) follows.

As a consequence, we have the following corollary.

Corollary 3.1 Under the same assumptions of Theorem 3.1 and for $k$ small enough, there exists a constant $c>0$ such that

$$
\begin{aligned}
& \left\|\partial_{x_{0}} u\right\|+\sum_{j=1}^{m}\left\|\partial_{x_{j}} u\right\|+\sum_{j=m+1}^{n}\left\|\left(x_{0}+\lambda-\alpha\left(x^{\prime}\right)\right) \partial_{x_{j}} u\right\|+\|u\| \leq c\|P u\|, \\
& \forall u \in C_{0}^{\infty}\left(\bar{\Omega}_{k}\right) .
\end{aligned}
$$

Proof Taking into account (4) and (7) and choosing a positive number $k$ small enough, we obtain (21).

\section{Estimates in Sobolev spaces with $s<0$ by means of pseudodifferential operator theory}

Let us, first, prove some preliminary results.

Lemma 4.1 Let $u \in C_{0}^{\infty}(\bar{\Omega})$, with $\bar{\Omega}=\left[0,+\infty\left[\times \Omega^{\prime} \times \mathbb{R}^{n-m}\right.\right.$, and let $\varphi \in C_{0}^{\infty}\left(\mathbb{R}^{n-m}\right)$, with $\operatorname{supp} \varphi \subseteq \mathbb{R}^{n-m} \backslash \mathcal{U}_{x^{\prime \prime}}$. As a result

$$
\left\|\varphi A_{s} u\right\|_{L^{2}(\bar{\Omega})} \leq \frac{c_{q, r, s}}{L^{q}}\|u\|_{H^{0,0, r}(\bar{\Omega})}, \quad \forall s \in \mathbb{R}, r \in \mathbb{Z}^{-}, q \geq s+r,
$$

where $L$ is the distance between $\operatorname{supp} \varphi$ and $\mathcal{U}_{x^{\prime \prime}}$, supposed to be greater than 1.

Proof Let us consider

$$
\begin{aligned}
\varphi A_{s} u= & \frac{1}{(2 \pi)^{n-m}} \int_{\mathbb{R}^{n-m}} e^{i x^{\prime \prime} \cdot \xi^{\prime \prime}} \varphi\left(x^{\prime \prime}\right)\left(1+\left|\xi^{\prime \prime}\right|^{2}\right)^{\frac{1}{2}} \widehat{u}\left(x_{0}, x^{\prime}, \xi^{\prime \prime}\right) d \xi^{\prime \prime} \\
= & \frac{1}{(2 \pi)^{n-m}} \int_{\mathbb{R}^{2(n-m)}} e^{i\left(x^{\prime \prime}-y^{\prime \prime}\right) \cdot \xi^{\prime \prime}} \varphi\left(x^{\prime \prime}\right)\left(1+\left|\xi^{\prime \prime}\right|^{2}\right)^{\frac{1}{2}} u\left(x_{0}, x^{\prime}, y^{\prime \prime}\right) d y^{\prime \prime} d \xi^{\prime \prime} \\
= & \frac{i^{2 p}}{(2 \pi)^{m-n}} \int_{\mathbb{R}^{2(n-m)}} e^{i\left(x^{\prime \prime}-y^{\prime \prime}\right) \cdot \xi^{\prime \prime}} \frac{\varphi\left(x^{\prime \prime}\right) u\left(x_{0}, x^{\prime}, y^{\prime \prime}\right)}{\left|x^{\prime \prime}-y^{\prime \prime}\right|^{2 p}}\left({\xi^{\prime \prime}}^{\prime \prime}\left(1+\left|\xi^{\prime \prime}\right|^{2}\right)^{\frac{s}{2}}\right)^{[p]} d y^{\prime \prime} d \xi^{\prime \prime} \\
= & \frac{i^{2 p}}{(2 \pi)^{m-n}} \int_{\mathbb{R}^{n-m}}\left(\triangle_{\xi^{\prime \prime}}\left(1+\left|\xi^{\prime \prime}\right|^{2}\right)^{\frac{s}{2}}\right)^{[p]} d \xi^{\prime \prime} \\
& \times \int_{\mathbb{R}^{n-m}} e^{i\left(x^{\prime \prime}-y^{\prime \prime}\right) \cdot \xi^{\prime \prime}} u\left(x_{0}, x^{\prime}, y^{\prime \prime}\right) \frac{\psi\left(\frac{\left|x^{\prime \prime}-y^{\prime \prime}\right|}{L}\right) \varphi\left(x^{\prime \prime}\right)}{\left|x^{\prime \prime}-y^{\prime \prime}\right|^{2 p}} d y^{\prime \prime},
\end{aligned}
$$

where $m \in \mathbb{N}$ and $\psi \in C^{\infty}(\mathbb{R})$ such that $\psi(\tau)=1$ if $|\tau| \geq 1, \psi(\tau)=0$ if $|\tau| \leq \frac{1}{2}$. 
This implies

$$
\varphi A_{s} u=\frac{i^{2 p} \varphi\left(x^{\prime \prime}\right)}{(2 \pi)^{n-m}} \int_{\mathbb{R}^{n-m}}\left(\triangle_{\xi^{\prime \prime}}\left(1+\left|\xi^{\prime \prime}\right|^{2}\right)^{\frac{s}{2}}\right)^{[p]} u\left(x_{0}, x^{\prime}, x^{\prime \prime}\right) * \psi\left(\frac{\left|x^{\prime \prime}\right|}{L}\right) \frac{e^{i x^{\prime \prime} \cdot \xi^{\prime \prime}}}{\left|x^{\prime \prime}\right|^{2 p}} d \xi^{\prime \prime}
$$

where the convolution is done with respect to $x^{\prime \prime}$, and also

$$
\begin{aligned}
\mathcal{F}_{x^{\prime \prime}}\left(\varphi A_{s} u\right)= & \frac{i^{2 p} \widehat{\varphi}\left(\eta^{\prime \prime}\right)}{2 \pi} \\
& * \int_{\mathbb{R}^{n-m}}\left(\triangle_{\xi^{\prime \prime}}\left(1+\left|\xi^{\prime \prime}\right|^{2}\right)^{\frac{s}{2}}\right)^{[p]} \widehat{u}\left(x_{0}, x^{\prime}, \eta^{\prime \prime}\right) \mathcal{F}_{x^{\prime \prime}}\left(\psi\left(\frac{\left|x^{\prime \prime}\right|}{L}\right) \frac{e^{i x^{\prime \prime} \cdot \xi^{\prime \prime}}}{\left|x^{\prime \prime}\right|^{2 p}}\right) d \xi^{\prime \prime}
\end{aligned}
$$

where

$$
\mathcal{F}_{x^{\prime \prime}}\left(\psi\left(\frac{\left|x^{\prime \prime}\right|}{L}\right) \frac{e^{i x^{\prime \prime} \cdot \xi^{\prime \prime}}}{\left|x^{\prime \prime}\right|^{2 p}}\right)=\int_{\mathbb{R}^{n-m}} e^{i x^{\prime \prime} \cdot\left(\xi^{\prime \prime}-\eta^{\prime \prime}\right)} \psi\left(\frac{\left|x^{\prime \prime}\right|}{L}\right) \frac{1}{\left|x^{\prime \prime}\right|^{2 p}} d x^{\prime \prime}
$$

It results

$$
\begin{aligned}
(1+ & \left.\left|\xi^{\prime \prime}-\eta^{\prime \prime}\right|^{2 r^{\prime}}\right) \mathcal{F}_{x^{\prime \prime}}\left(\psi\left(\frac{\left|x^{\prime \prime}\right|}{L}\right) \frac{e^{i x^{\prime \prime} \cdot \xi^{\prime \prime}}}{\left|x^{\prime \prime}\right|^{2 p}}\right) \\
= & \int_{\mathbb{R}^{n-m}} e^{i x^{\prime \prime} \cdot\left(\xi^{\prime \prime}-\eta^{\prime \prime}\right)} \psi\left(\frac{\left|x^{\prime \prime}\right|}{L}\right) \frac{1}{\left|x^{\prime \prime}\right|^{2 p}} d x^{\prime \prime} \\
& +(-1)^{r} \int_{\mathbb{R}^{n-m}} e^{i x^{\prime \prime} \cdot\left(\xi^{\prime \prime}-\eta^{\prime \prime}\right)}\left(\triangle_{x^{\prime \prime}}\left(\psi\left(\frac{\left|x^{\prime \prime}\right|}{L}\right) \frac{1}{\left|x^{\prime \prime}\right|^{2 p}}\right)\right)^{[r]} d x^{\prime \prime}
\end{aligned}
$$

and then

$$
\left|\mathcal{F}_{x^{\prime \prime}}\left(\psi\left(\frac{\left|x^{\prime \prime}\right|}{L}\right) \frac{e^{i x^{\prime \prime} \cdot \xi^{\prime \prime}}}{\left|x^{\prime \prime}\right|^{2 p}}\right)\right| \leq \frac{c_{r, p}}{\left(1+\left|\xi^{\prime \prime}-\eta^{\prime \prime}\right|^{2 r^{\prime}}\right)}\left(\frac{1}{L}\right)^{2 p-n+m+1} .
$$

Making use of (22) and (23), we obtain

$$
\begin{aligned}
&\left\|\mathcal{F}_{x^{\prime \prime}}\left(\varphi A_{s} u\right)\right\| \\
&=\left\|\varphi A_{s} u\right\| \\
& \leq \frac{1}{(2 \pi)^{n-m}}\left\|\widehat{\varphi}\left(\eta^{\prime \prime}\right)\right\|_{L^{1}\left(\mathbb{R}^{n-m}\right)} \\
& \cdot\left\|\int_{\mathbb{R}^{n-m}}\left(\triangle_{\xi^{\prime \prime}}\left(1+\left|\xi^{\prime \prime}\right|^{2}\right)^{\frac{s}{2}}\right)^{[p]} \widehat{u}\left(x_{0}, x^{\prime}, \eta^{\prime \prime}\right) \mathcal{F}_{x^{\prime \prime}}\left(\psi\left(\frac{\left|x^{\prime \prime}\right|}{L}\right) \frac{e^{i x^{\prime \prime} \cdot \xi^{\prime \prime}}}{\left|x^{\prime \prime}\right|^{2 p}}\right) d \xi^{\prime \prime}\right\|_{L^{2}(\Omega)} \\
& \leq c \int_{\mathbb{R}^{n-m}}\left\|\left(\triangle_{\xi^{\prime \prime}}\left(1+\left|\xi^{\prime \prime}\right|^{2}\right)^{\frac{s}{2}}\right)^{[p]} \widehat{u}\left(x_{0}, x^{\prime}, \eta^{\prime \prime}\right) \mathcal{F}_{x^{\prime \prime}}\left(\psi\left(\frac{\left|x^{\prime \prime}\right|}{L}\right) \frac{e^{i x^{\prime \prime} \cdot \xi^{\prime \prime}}}{\left|x^{\prime \prime}\right|^{2 p}}\right)\right\|_{L^{2}(\Omega)} d \xi^{\prime \prime} \\
& \leq \frac{c_{r, p}}{L^{2 p-n+m+1}} \int_{\mathbb{R}^{n-m}}\left\|\frac{\left(\triangle_{\xi^{\prime \prime}}\left(1+\left|\xi^{\prime \prime}\right|^{2}\right)^{\frac{s}{2}}\right)^{[p]} \widehat{u}\left(x_{0}, x^{\prime}, \eta^{\prime \prime}\right)}{\left(1+\left|\xi^{\prime \prime}-\eta^{\prime \prime}\right|^{\left.2 r^{\prime}\right)}\right.}\right\|_{L^{2}(\Omega)} d \xi^{\prime \prime} .
\end{aligned}
$$

Taking into account the previous inequality and the Peetre inequality (see [16], p. 17), it follows that

$$
\left\|\varphi A_{s} u\right\|_{L^{2}(\bar{\Omega})} \leq \frac{c_{r, p, s}}{L^{2 p-n+m}} \int_{\mathbb{R}^{n-m}} \frac{\left\|\left(1+\left|\xi^{\prime \prime}\right|^{2}\right)^{\frac{s}{2}-\frac{2 p+n-m}{2}} \widehat{u}\left(x_{0}, x^{\prime}, \eta^{\prime \prime}\right)\right\|_{L^{2}(\Omega)}}{\left(1+\left|\xi^{\prime \prime}-\eta^{\prime \prime}\right|^{2 r}\right)} d \xi^{\prime \prime} .
$$


If $p \geq \frac{s+r+n-m+1}{2}$, setting $q=2 p-n+m+1$ in (24), $r=2 r^{\prime}$, results in

$$
\left\|\varphi A_{s} u\right\|_{L^{2}(\Omega)} \leq \frac{c_{q, r, s}}{L^{q}}\|u\|_{H^{0,0, r}(\Omega)},
$$

where the constant $c_{q, r, s}$ is independent on $L$.

Taking into account Lemma 4.1, it is easy to show the following.

Lemma 4.2 Let $\varphi \in C_{0}^{\infty}\left(\mathbb{R}^{n-m}\right)$ such that $\varphi\left(\left|\tau^{\prime \prime}\right|\right)=0$ if $\left|\tau^{\prime \prime}\right| \leq 1$. For every $\varepsilon>0, r \in \mathbb{Z}^{-}$, $s \in \mathbb{R}$ and for every $u \in C_{0}^{\infty}(\bar{\Omega})$, with $\bar{\Omega}=\left[0,+\infty\left[\times \Omega^{\prime} \times \mathbb{R}^{n-m}\right.\right.$, there exists $L>0$ such that

$$
\left\|\varphi\left(\frac{\left|x^{\prime \prime}\right|}{L}\right) A_{s} u\right\|_{L^{2}(\Omega)} \leq \varepsilon\|u\|_{H^{0,0, r}(\Omega)} .
$$

Furthermore, we are able to prove the following.

Lemma 4.3 Let $\varphi \in C_{0}^{\infty}\left(\mathbb{R}^{n-m}\right)$ such that $\varphi\left(\left|\tau^{\prime \prime}\right|\right)=1$ if $\left|\tau^{\prime \prime}\right| \leq 1$. For every $\varepsilon>0, r \in \mathbb{Z}^{-}$, $s \in \mathbb{R}$ and for every $u \in C_{0}^{\infty}(\bar{\Omega})$, with $\bar{\Omega}=\left[0,+\infty\left[\times \Omega^{\prime} \times \mathbb{R}^{n-m}\right.\right.$, there exists $L>0$ such that

$$
\left\|\left(1-\varphi\left(\frac{\left|x^{\prime \prime}\right|}{L}\right)\right) A_{s} u\right\|_{L^{2}(\Omega)} \leq \varepsilon\|u\|_{H^{0,0, r}(\Omega)} .
$$

Proof In order to establish this result we can proceed as Lemma 4.1, but in (22) we need to consider the Fourier transform of the function $\psi\left(\left|x^{\prime \prime}\right|\right)=1-\varphi\left(\frac{\left|x^{\prime \prime}\right|}{L}\right)$ instead of $\widehat{\varphi}\left(\eta^{\prime \prime}\right)$ and keep in mind that

$$
\widehat{\psi g}=\widehat{\psi} * \widehat{g}=(2 \pi \delta-\widehat{\varphi}) * \widehat{g}=2 \pi \widehat{g}-\widehat{\varphi} * \widehat{g}, \quad \forall g \in S^{\prime}(\mathbb{R})
$$

where $S^{\prime}(\mathbb{R})$ is the space of tempered distributions defined in $\mathbb{R}$.

Next, we prove a result concerning estimates near the boundary.

Lemma 4.4 Let $\Omega=] 0,+\infty\left[\times \Omega_{0}\right.$, where $\Omega_{0}$ is an open subset of $\mathbb{R}^{n}$. For every $\varepsilon$ and $\delta$ positive, there exists $k>0$ such that if

$$
I_{k, \delta}=\left\{x \in \bar{\Omega}: x_{0}<k,\left|x_{0}+\lambda-\alpha\left(x^{\prime}\right)\right|>\delta\right\}
$$

as a result

$$
\begin{gathered}
\left\|\partial_{x_{0}} u\right\|+\sum_{j=1}^{m}\left\|\partial_{x_{j}} u\right\|+\sum_{j=m+1}^{n}\left\|\left(x_{0}+\lambda-\alpha\left(x^{\prime}\right)\right) \partial_{x_{j}} u\right\|+\|u\| \leq \varepsilon\|P u\|, \\
\forall u \in C_{0}^{\infty}(\bar{\Omega}): \operatorname{supp} u \subseteq I_{k, \delta} .
\end{gathered}
$$

Proof Integrating by parts and proceeding as in the first part of Theorem 3.1, we have

$$
\begin{aligned}
2\left(e^{\tau x_{0}} \partial_{x_{0}} u(x), P u\right)= & \tau\left\|e^{\frac{1}{2} \tau x_{0}} \partial_{x_{0}} u\right\|^{2}+\int_{\Omega_{0}}\left(\partial_{x_{0}} u\left(0, x^{\prime}, x^{\prime \prime}\right)\right)^{2} d x^{\prime} d x^{\prime \prime} \\
& +\int_{\Omega} e^{\tau x_{0}} A\left(x^{\prime}, x^{\prime \prime}\right) \nabla_{x^{\prime}} u(x) \cdot \nabla_{x^{\prime}} u(x) d x
\end{aligned}
$$




$$
\begin{aligned}
& +\int_{\Omega_{0}} A\left(x^{\prime}, x^{\prime \prime}\right) \nabla_{x^{\prime}} u\left(0, x^{\prime}, x^{\prime \prime}\right) \cdot \nabla_{x^{\prime}} u\left(0, x^{\prime}, x^{\prime \prime}\right) d x^{\prime} d x^{\prime \prime} \\
& +2 \int_{\Omega} e^{\tau x_{0}}\left(x_{0}+\lambda-\alpha\left(x^{\prime}\right)\right) B\left(x^{\prime \prime}\right) \nabla_{x^{\prime \prime}} u(x) \cdot \nabla_{x^{\prime \prime}} u(x) d x \\
& +\tau \int_{\Omega} e^{\tau x_{0}}\left(x_{0}+\lambda-\alpha\left(x^{\prime}\right)\right)^{2} B\left(x^{\prime \prime}\right) \nabla_{x^{\prime \prime}} u(x) \cdot \nabla_{x^{\prime \prime}} u(x) d x \\
& +\lambda \int_{\Omega_{0}}\left(\lambda-\alpha\left(x^{\prime}\right)\right)^{2} B\left(x^{\prime \prime}\right) \nabla_{x^{\prime \prime}} u\left(0, x^{\prime}, x^{\prime \prime}\right) \cdot \nabla_{x^{\prime \prime}} u\left(0, x^{\prime}, x^{\prime \prime}\right) d x^{\prime} d x^{\prime \prime} \\
& -\int_{\Omega} \tau e^{\tau x_{0}} \gamma(x) u^{2}(x) d x-\int_{\Omega} e^{\tau x_{0}} u^{2}(x) \partial_{x_{0}} \gamma(x) d x \\
& -\int_{\Omega_{0}} \gamma\left(0, x^{\prime}, x^{\prime \prime}\right) u^{2}\left(0, x^{\prime}, x^{\prime \prime}\right) d x^{\prime} d x^{\prime \prime}
\end{aligned}
$$

This implies

$$
\begin{aligned}
& \tau\left(\left\|e^{\frac{1}{2} \tau x_{0}} \partial_{x_{0}} u\right\|^{2}+L_{1} \sum_{j=1}^{m}\left\|e^{\frac{1}{2} \tau x_{0}} \partial_{x_{j}} u\right\|^{2}+L_{2} \sum_{j=m+1}^{n}\left\|\left(x_{0}+\lambda-\alpha\left(x^{\prime}\right)\right) e^{\frac{1}{2} \tau x_{0}} \partial_{x_{j}} u\right\|^{2}\right) \\
& \leq c \sum_{j=m+1}^{n}\left\|\frac{\left(x_{0}+\lambda-\alpha\left(x^{\prime}\right)\right)}{\left(x_{0}+\lambda-\alpha\left(x^{\prime}\right)\right)^{\frac{1}{2}}} e^{\frac{1}{2} \tau x_{0}} \partial_{x_{j}} u\right\|^{2}+\tau\left\|e^{\frac{1}{2} \tau x_{0}}|\gamma(x)|^{\frac{1}{2}} u(x)\right\|^{2} \\
& \quad+\left\|e^{\frac{1}{2} \tau x_{0}}\left|\partial_{x_{0}} \gamma(x)\right|^{\frac{1}{2}} u(x)\right\|^{2}+\int_{\Omega_{0}}\left|\gamma\left(0, x^{\prime}, x^{\prime \prime}\right)\right| u^{2}\left(0, x^{\prime}, x^{\prime \prime}\right) d x^{\prime} d x^{\prime \prime} \\
& \quad+\left(c e^{\tau x_{0}} P u, \partial_{x_{0}} u\right) \\
& \leq \frac{c}{\delta} \sum_{j=m+1}^{n}\left\|e^{\frac{1}{2} \tau x_{0}}\left(x_{0}+\lambda-\alpha\left(x^{\prime}\right)\right) \partial_{x_{j}} u\right\|^{2}+c\left(\frac{1}{\tau^{2}}+\frac{1}{\tau}\right)\left\|\partial_{x_{0}} u\right\|^{2}+\frac{1}{2}\left\|e^{\frac{1}{2} \tau x_{0}} P u\right\|^{2} \\
& \quad+\frac{1}{2}\left\|e^{\frac{1}{2} \tau x_{0}} \partial_{x_{0}} u\right\|^{2} .
\end{aligned}
$$

Choosing $x_{0}<\frac{1}{\tau}$, it follows that

$$
\begin{aligned}
& \left\|\partial_{x_{0}} u\right\|^{2}+\sum_{j=1}^{m}\left\|\partial_{x_{j}} u\right\|^{2}+\sum_{j=m+1}^{n}\left\|\left(x_{0}+\lambda-\alpha\left(x^{\prime}\right)\right) \partial_{x_{j}} u\right\|^{2} \\
& \quad \leq \frac{c}{\tau \delta} \sum_{j=m+1}^{n}\left\|e^{\frac{1}{2} \tau x_{0}}\left(x_{0}+\lambda-\alpha\left(x^{\prime}\right)\right) \partial_{x_{j}} u\right\|^{2}+c \frac{1}{\tau}\|P u\|^{2}+\frac{c}{\tau}\left\|\partial_{x_{0}} u\right\|^{2} .
\end{aligned}
$$

For $\tau$ large enough, making use of (27) and (4) we obtain the claim.

As a consequence, we establish the following result.

Lemma 4.5 For every $\varepsilon$ and $\delta$ positive, there exists $k>0$ such that if

$$
I_{k, \delta}=\left\{x \in \bar{\Omega}: x_{0}<k,\left|x_{0}+\lambda-\alpha\left(x^{\prime}\right)\right|>\delta\right\}
$$


for every $s<0$, as a result

$$
\begin{aligned}
& \left\|\partial_{x_{0}} u\right\|_{H^{0,0, s}(\Omega)}+\sum_{j=1}^{m}\left\|\partial_{x_{j}} u\right\|_{H^{0,0, s}(\Omega)}+\sum_{j=m+1}^{n}\left\|\left(x_{0}+\lambda-\alpha\left(x^{\prime}\right)\right) \partial_{x_{j}} u\right\|_{H^{0,0, s}(\Omega)}+\|u\|_{H^{0,0, s}(\Omega)} \\
& \leq \varepsilon\|P u\|_{H^{0,0, s}(\Omega)}, \quad \forall u \in C_{0}^{\infty}(\bar{\Omega}): \operatorname{supp} u \subseteq I_{k, \delta} .
\end{aligned}
$$

Proof Let $\varphi \in C_{0}^{\infty}(\mathbb{R})$, set $v_{s}=\varphi\left(\left|x^{\prime \prime}\right|\right) A_{s} u$, as a result $\operatorname{supp} v_{s} \subseteq I_{k, \delta}$ and $\varphi\left(\left|x^{\prime \prime}\right|\right)=1$ in $\mathcal{U}_{x^{\prime \prime}}$. Therefore we can rewrite (25) with $v_{s}$, namely

$$
\left\|\partial_{x_{0}} v_{s}\right\|+\sum_{j=1}^{m}\left\|\partial_{x_{j}} v_{s}\right\|+\sum_{j=m+1}^{n}\left\|\left(x_{0}+\lambda-\alpha\left(x^{\prime}\right)\right) \partial_{x_{j}} v_{s}\right\|+\left\|v_{s}\right\| \leq \varepsilon\left\|P v_{s}\right\|
$$

Let us compute

$$
\begin{aligned}
\left\|\partial_{x_{0}} v_{s}\right\| & \geq\left\|\partial_{x_{0}} A_{s} u\right\|-\left\|(\varphi-1) \partial_{x_{0}} A_{s} u\right\| \\
& =\left\|\partial_{x_{0}} A_{s} u\right\|-\|R u\|,
\end{aligned}
$$

where $(\varphi-1) \partial_{x_{0}} A_{s}=R$ is a regularizing operator. In the same way,

$$
\left\|\partial_{x_{j}} v_{s}\right\| \geq c\left\|A_{s} \partial_{x_{j}} u\right\|-\|R u\|, \quad \forall j=1, \ldots, m
$$

Similarly, we obtain

$$
\begin{aligned}
\left\|\left(x_{0}+\lambda-\alpha\left(x^{\prime}\right)\right) \partial_{x_{j}} v_{s}\right\|= & \left\|\left(x_{0}+\lambda-\alpha\left(x^{\prime}\right)\right) \partial_{x_{j}} \varphi A_{s} u\right\| \\
= & \| A_{s}\left(x_{0}+\lambda-\alpha\left(x^{\prime}\right)\right) \partial_{x_{j}} u+(\varphi-1) A_{s}\left(x_{0}+\lambda-\alpha\left(x^{\prime}\right)\right) \partial_{x_{j}} u \\
& +\left(x_{0}+\lambda-\alpha\left(x^{\prime}\right)\right)\left[\partial_{x_{j}}, \varphi\right]\left(A_{s} u\right) \| \\
\geq & \left\|A_{s}\left(x_{0}+\lambda-\alpha\left(x^{\prime}\right)\right) \partial_{x_{j}} u\right\|-c\|R u\|,
\end{aligned}
$$

where we take into account that $(\varphi-1) A_{s} \partial_{x_{j}}$ and $\left[\partial_{x_{j}}, \varphi\right] A_{s}$ are regularizing operators. Finally, we have

$$
\begin{aligned}
\left\|v_{s}\right\| & =\left\|A_{s} u+(\varphi-1) A_{s} u\right\| \\
& \geq\left\|A_{s} u\right\|-\|R u\|,
\end{aligned}
$$

$(\varphi-1) A_{s}$ being a regularizing operator.

Making use of (30), (31), (32), and (33), it follows that

$$
\begin{aligned}
& \left\|\partial_{x_{0}} v_{s}\right\|+\sum_{j=1}^{m}\left\|\partial_{x_{j}} v_{s}\right\|+\sum_{j=m+1}^{n}\left\|\left(x_{0}+\lambda-\alpha\left(x^{\prime}\right)\right) \partial_{x_{j}} v_{s}\right\|+\left\|v_{s}\right\| \\
& \geq\left\|\partial_{x_{0}} u\right\|_{H^{0,0, s}\left(\Omega_{k}\right)}+c\left(\sum_{j=1}^{m}\left\|\partial_{x_{j}} u\right\|_{H^{0,0, s}\left(\Omega_{k}\right)}+\sum_{j=m+1}^{n}\left\|\left(x_{0}+\lambda-\alpha\left(x^{\prime}\right)\right) \partial_{x_{j}} u\right\|_{H^{0,0, s}\left(\Omega_{k}\right)}\right) \\
& \quad+\|u\|_{H^{0,0, s}\left(\Omega_{k}\right)}-c\|R u\| .
\end{aligned}
$$


Since $\|R u\| \leq c\|u\|_{H^{0,0, s}\left(\Omega_{k}\right)} \leq c k\left\|\partial_{x_{0}} u\right\|_{H^{0,0, s}\left(\Omega_{k}\right)}$, choosing $k$ small enough, as a result

$$
\begin{aligned}
& \left\|\partial_{x_{0}} v_{s}\right\|+\sum_{j=1}^{m}\left\|\partial_{x_{j}} v_{s}\right\|+\sum_{j=m+1}^{n}\left\|\left(x_{0}+\lambda-\alpha\left(x^{\prime}\right)\right) \partial_{x_{j}} v_{s}\right\|+\left\|v_{s}\right\| \\
& \geq c\left(\left\|\partial_{x_{0}} u\right\|_{H^{0,0, s}\left(\Omega_{k}\right)}+\sum_{j=1}^{m}\left\|\partial_{x_{j}} u\right\|_{H^{0,0, s}\left(\Omega_{k}\right)}+\sum_{j=m+1}^{n}\left\|\left(x_{0}+\lambda-\alpha\left(x^{\prime}\right)\right) \partial_{x_{j}} u\right\|_{H^{0,0, s}\left(\Omega_{k}\right)}\right. \\
& \left.\quad+\|u\|_{H^{0,0, s}\left(\Omega_{k}\right)}\right) .
\end{aligned}
$$

On the other hand, we have

$$
P v_{s}=\varphi\left(\left|x^{\prime \prime}\right|\right) A_{s} P u+\varphi\left(\left|x^{\prime \prime}\right|\right)\left[P, A_{s}\right](u)+\left[\varphi\left(\left|x^{\prime \prime}\right|\right), P\right]\left(A_{s} u\right) .
$$

As a consequence, we obtain

$$
\left\|P v_{s}\right\|=\left\|\varphi\left(\left|x^{\prime \prime}\right|\right) A_{s} P u+\varphi\left(\left|x^{\prime \prime}\right|\right) B_{s+1} u+R u\right\|,
$$

where we set $\left[P, A_{s}\right]=B_{s+1}$, this being a pseudodifferential operator endowed with the symbol with respect to the variable $x^{\prime \prime}$ of order $s+1$. Such a symbol has the following principal part:

$$
c\left(x, \xi^{\prime \prime}\right)=-\frac{1}{i} \sum_{p=m+1}^{n}\left(x_{0}+\lambda-\alpha\left(x^{\prime}\right)\right)^{2} \sum_{i, j=m+1}^{n} \partial_{x_{p}} b_{j}\left(x^{\prime \prime}\right) \xi_{i}^{\prime \prime} \xi_{j}^{\prime \prime} \partial_{\xi_{p}}\left(1+\left|\xi^{\prime \prime}\right|^{2}\right)^{\frac{s}{2}}
$$

Therefore the symbol $b\left(x, \xi^{\prime \prime}\right)$ can be written as

$$
b\left(x, \xi^{\prime \prime}\right)=c\left(x, \xi^{\prime \prime}\right)+d\left(x, \xi^{\prime \prime}\right)
$$

where $d\left(x, \xi^{\prime \prime}\right)$ is a symbol of order $s$ and we set

$$
B_{s+1}=C_{s+1}+D_{s}
$$

Moreover, we set $R=\left[\varphi\left(\left|x^{\prime \prime}\right|\right), P\right] A_{s}$, which is a regularizing operator.

At last, we remark that

$$
C_{s+1} u=C_{s}^{\prime}\left(x_{0}+\lambda-\alpha\left(x^{\prime}\right)\right) \sum_{i=m+1}^{n} \partial_{x_{j}} u
$$

and the symbol $c_{s}^{\prime}$ of $C_{s}^{\prime}$ is given by

$$
c_{s}^{\prime}\left(x, \xi^{\prime \prime}\right)=-\frac{1}{i} \sum_{p=m+1}^{n}\left(x_{0}+\lambda-\alpha\left(x^{\prime}\right)\right) \sum_{j=m+1}^{n} \partial_{x_{p}} b_{j}\left(x^{\prime \prime}\right) \xi_{j}^{\prime \prime} \partial_{\xi_{p}}\left(1+\left|\xi^{\prime \prime}\right|^{2}\right)^{\frac{s}{2}} .
$$


By such insights and by (35), as a result

$$
\begin{aligned}
\left\|P v_{s}\right\| & \leq c\left(\left\|A_{s} P u\right\|+\sum_{j=m+1}^{n}\left\|C_{s}^{\prime}\left(x_{0}+\lambda-\alpha\left(x^{\prime}\right)\right) \partial_{x_{j}} u\right\|+\left\|D_{s} u\right\|+\|R u\|\right) \\
& \leq c\left(\|P u\|_{H^{0,0, s}(\Omega)}+\sum_{j=m+1}^{n}\left\|\left(x_{0}+\lambda-\alpha\left(x^{\prime}\right)\right) \partial_{x_{j}} u\right\|_{H^{0,0, s}(\Omega)}+\|u\|_{H^{0,0, s}(\Omega)}\right) .
\end{aligned}
$$

By using (29), (34), (36), and for $\varepsilon$ small enough, the claim follows.

Now, we are able to prove the following theorem.

Theorem 4.1 Let $\Omega_{k}=\left[0, k\left[\times \Omega_{0}\right.\right.$, with $k$ such that $(21)$ holds, let $\Omega_{0}=\Omega^{\prime} \times \mathbb{R}^{n-m}$, let $\Omega^{\prime}$ be an open set of $\mathbb{R}^{n-m}$ and let $B\left(x^{\prime \prime}\right)$ be a constant. Under assumptions (i) and (ii), for every $s \in \mathbb{Z}^{-}$there exists $c>0$ such that

$$
\begin{aligned}
& \left\|\partial_{x_{0}} u\right\|_{H^{0,0, s}(\Omega)}+\sum_{j=1}^{m}\left\|\partial_{x_{j}} u\right\|_{H^{0,0, s}(\Omega)}+\sum_{j=m+1}^{n}\left\|\left(x_{0}+\lambda-\alpha\left(x^{\prime}\right)\right) \partial_{x_{j}} u\right\|_{H^{0,0, s}(\Omega)}+\|u\|_{H^{0,0, s}(\Omega)} \\
& \leq c\|P u\|_{H^{0,0, s}\left(\Omega_{k}\right)}, \quad \forall u \in C_{0}^{\infty}(\bar{\Omega}): \operatorname{supp} u \subseteq \Omega_{k} .
\end{aligned}
$$

Proof Let $\varphi \in C_{0}^{\infty}(\mathbb{R})$ and $\varphi\left(\tau^{\prime}\right)=1$ if $\left|\tau^{\prime}\right| \leq 1$ and $\mathcal{U}_{x^{\prime \prime}} \subseteq[-L, L]^{n-m}$. Setting $v_{s}=\varphi\left(\frac{\left|x^{\prime \prime}\right|}{L}\right) A_{s} u$ in (21), as a result

$$
\left\|\partial_{x_{0}} v_{s}\right\|+\sum_{j=1}^{m}\left\|\partial_{x_{j}} v_{s}\right\|+\sum_{j=m+1}^{n}\left\|\left(x_{0}+\lambda-\alpha\left(x^{\prime}\right)\right) \partial_{x_{j}} v_{s}\right\|+\left\|v_{s}\right\| \leq c\left\|P v_{s}\right\| .
$$

Furthermore, we have

$$
\begin{aligned}
\left\|\partial_{x_{0}} v_{s}\right\| & =\left\|\partial_{x_{0}} A_{s} u+(\varphi-1) \partial_{x_{0}} A_{s} u\right\| \\
& \geq\left\|A_{s} \partial_{x_{0}} u\right\|-\left\|(\varphi-1) A_{s} \partial_{x_{0}} u\right\| .
\end{aligned}
$$

Taking into account Lemma 4.3, for $L$ large enough, it follows that

$$
\left\|\partial_{x_{0}} v_{s}\right\| \geq c\left\|A_{s} \partial_{x_{0}} u\right\|
$$

Making use of the same technique, we have

$$
\left\|\partial_{x_{j}} v_{s}\right\| \geq c\left\|A_{s} \partial_{x_{j}} u\right\|, \quad \forall j=1, \ldots, m,
$$

and similarly

$$
\begin{aligned}
\left\|\left(x_{0}+\lambda-\alpha\left(x^{\prime}\right)\right) \partial_{x_{j}} v_{s}\right\| & =\| A_{s}\left(x_{0}+\lambda-\alpha\left(x^{\prime}\right)\right) \partial_{x_{j}} u+(\varphi-1) A_{s}\left(x_{0}+\lambda-\alpha\left(x^{\prime}\right)\right) \partial_{x_{j}} u \\
& +\left[\partial_{x_{j}}, \varphi\left(\frac{\left|x^{\prime \prime}\right|}{L}\right)\right] A_{s}\left(x_{0}+\lambda-\alpha\left(x^{\prime}\right)\right) u \| \\
\geq & c\left\|A_{s}\left(x_{0}+\lambda-\alpha\left(x^{\prime}\right)\right) \partial_{x_{j}} u\right\|-\frac{c}{L}\left\|A_{s}\left(x_{0}+\lambda-\alpha\left(x^{\prime}\right)\right) u\right\|,
\end{aligned}
$$


where $L$ is large enough. Finally, we have

$$
\begin{aligned}
\left\|v_{s}\right\| & =\left\|A_{s} u\right\|+\left\|\left(1-\varphi\left(\frac{\left|x^{\prime \prime}\right|}{L}\right)\right) A_{s} u\right\| \\
& \geq(1-\varepsilon)\|u\|_{H^{0,0, s}(\Omega)},
\end{aligned}
$$

having used Lemma 4.2 .

On the other hand we have the result

$$
P v_{s}=\varphi\left(\frac{\left|x^{\prime \prime}\right|}{L}\right) A_{s} P u+\left[\varphi\left(\frac{\left|x^{\prime \prime}\right|}{L}\right), P\right]\left(A_{s} u\right)
$$

Let us observe that

$$
\left\|A_{s} P u\right\|=\|P u\|_{H^{0,0, s}(\Omega)},
$$

from the continuity property of the pseudodifferential operators (see [16], Theorem 2.1) and making use of Lemma 4.2

$$
\left\|\left[\varphi\left(\frac{\left|x^{\prime \prime}\right|}{L}\right), P\right]\left(A_{s} u\right)\right\| \leq \varepsilon\|u\|_{H^{0,0, s}(\Omega)} .
$$

By using (43), (44), and (45), we have

$$
\begin{aligned}
\left\|P v_{s}\right\| & \leq\left\|\varphi\left(\frac{\left|x^{\prime \prime}\right|}{L}\right) A_{s} P u\right\|+\left\|\varphi\left(\frac{\left|x^{\prime \prime}\right|}{L}\right) B_{s} u\right\|+\left\|\left[\varphi\left(\frac{\left|x^{\prime \prime}\right|}{L}\right), P\right]\left(A_{s} u\right)\right\| \\
& \leq c\|P u\|_{H^{0,0, s}(\Omega)}+\varepsilon\|u\|_{H^{0,0, s}(\Omega)} .
\end{aligned}
$$

Making use of (38), (39), (40), (41), and (42), choosing $\varepsilon$ small enough and taking into account Lemma 3.1, as a result

$$
\begin{aligned}
& \left\|\partial_{x_{0}} u\right\|_{H^{0,0, s}(\Omega)}+\sum_{j=1}^{m}\left\|\partial_{x_{j}} u\right\|_{H^{0,0, s}(\Omega)}+\sum_{j=m+1}^{n}\left\|\left(x_{0}+\lambda-\alpha\left(x^{\prime}\right)\right) \partial_{x_{j}} u\right\|_{H^{0,0, s}(\Omega)}+\|u\|_{H^{0,0, s}(\Omega)} \\
& \quad \leq c\|P u\|_{H^{0,0, s}(\Omega)}+\varepsilon\|u\|_{H^{0,0, s}(\Omega)} \\
& \quad \leq c\|P u\|_{H^{0,0, s}(\Omega)}+\varepsilon\left\|\partial_{x_{0}} u\right\|_{H^{0,0, s}\left(\Omega_{k}\right)}
\end{aligned}
$$

and for $\varepsilon$ small enough the claim is established.

Now, we prove an estimate in Sobolev spaces with $s<0$.

Theorem 4.2 Under assumptions (i), (ii), (iii), and (iv), for every $s \in \mathbb{R}_{0}^{-}$there exists $c>0$ such that

$$
\begin{aligned}
& \left\|\partial_{x_{0}} u\right\|_{H^{0,0, s}(\Omega)}+\sum_{j=1}^{m}\left\|\partial_{x_{j}} u\right\|_{H^{0,0, s}(\Omega)}+\sum_{j=m+1}^{n}\left\|\left(x_{0}+\lambda-\alpha\left(x^{\prime}\right)\right) \partial_{x_{j}} u\right\|_{H^{0,0, s}(\Omega)}+\|u\|_{H^{0,0, s}(\Omega)} \\
& \leq c\|P u\|_{H^{0,0, s}\left(\Omega_{k}\right)}, \quad \forall u \in C_{0}^{\infty}(\bar{\Omega}): \operatorname{supp} u \subseteq \Omega_{k}=\left[0, k\left[\times \Omega_{0} .\right.\right.
\end{aligned}
$$


Proof Let $\varphi \in C_{0}^{\infty}(\mathbb{R})$ such that $\varphi\left(\left|x^{\prime \prime}\right|\right)=1$ on $\mathcal{U}_{x^{\prime \prime}}$ and let $k$ such that (21) holds.

Then, for every $u \in C_{0}^{\infty}(\bar{\Omega})$ such that $\operatorname{supp} u \subseteq \Omega_{k}$, as a result

$$
\left\|\partial_{x_{0}} v_{s}\right\|+\sum_{j=1}^{m}\left\|\partial_{x_{j}} v_{s}\right\|+\sum_{j=m+1}^{n}\left\|\left(x_{0}+\lambda-\alpha\left(x^{\prime}\right)\right) \partial_{x_{j}} v_{s}\right\|+\left\|v_{s}\right\| \leq c\left\|P v_{s}\right\|,
$$

where $v_{s}=\varphi\left(\left|x^{\prime \prime}\right|\right) A_{s} u$.

Proceeding as in the proof of (34) (see from (29) to (34)), we obtain

$$
\begin{aligned}
& \left\|\partial_{x_{0}} v_{s}\right\|+\sum_{j=1}^{m}\left\|\partial_{x_{j}} v_{s}\right\|+\sum_{j=m+1}^{n}\left\|\left(x_{0}+\lambda-\alpha\left(x^{\prime}\right)\right) \partial_{x_{j}} v_{s}\right\|+\left\|v_{s}\right\| \\
& \geq c\left(\left\|\partial_{x_{0}} u\right\|_{H^{0,0, s}\left(\Omega_{k}\right)}+\sum_{j=1}^{m}\left\|\partial_{x_{j}} u\right\|_{H^{0,0, s}\left(\Omega_{k}\right)}+\sum_{j=m+1}^{n}\left\|\left(x_{0}+\lambda-\alpha\left(x^{\prime}\right)\right) \partial_{x_{j}} u\right\|_{H^{0,0, s}\left(\Omega_{k}\right)}\right. \\
& \left.\quad+\|u\|_{H^{0,0, s}\left(\Omega_{k}\right)}\right)
\end{aligned}
$$

On the other hand, we have

$$
P v_{s}=\varphi\left(\left|x^{\prime \prime}\right|\right) A_{s} P u+\left[\varphi\left(\left|x^{\prime}\right|\right), P\right]\left(A_{s} u\right)+\varphi\left(\left|x^{\prime}\right|\right)\left[P, A_{s}\right] u=\varphi\left(\left|x^{\prime}\right|\right) A_{s} P u+R u+B_{s+1} u,
$$

where $R=\left[\varphi\left(\left|x^{\prime}\right|\right), P\right] A_{s}$ is a regularizing operator and $B_{s+1}=\left[P, A_{s}\right]$ is a pseudodifferential operator with respect to the variables $x^{\prime \prime}$ of order $s+1$ endowed with symbol $b\left(x, \xi^{\prime \prime}\right)$ with principal part equal to

$$
c\left(x, \xi^{\prime \prime}\right)=-\frac{1}{i} \sum_{p=m+1}^{n}\left(x_{0}+\lambda-\alpha\left(x^{\prime}\right)\right)^{2} \sum_{i, j=m+1}^{n} \partial_{x_{p}} b_{j}\left(x^{\prime \prime}\right) \xi_{i}^{\prime \prime} \xi_{j}^{\prime \prime} \partial_{\xi_{p}}\left(1+\left|\xi^{\prime \prime}\right|^{2}\right)^{\frac{s}{2}} .
$$

Hence,

$$
b\left(x, \xi^{\prime \prime}\right)=c\left(x, \xi^{\prime \prime}\right)+d\left(x, \xi^{\prime \prime}\right)
$$

where $c\left(x, \xi^{\prime \prime}\right)$ is the symbol of order $s$. Therefore, we have

$$
B_{s+1}=C_{s+1}+D_{s} .
$$

Then as a result

$$
\left[P, A_{s}\right](u)=C_{s+1} u+D_{s} u
$$

Taking into account (41), (42), (43), and (49), we obtain

$$
\begin{aligned}
\left\|P v_{s}\right\| & \leq\left\|\varphi\left(\frac{\left|x^{\prime \prime}\right|}{L}\right) A_{s} P u\right\|+\left\|\varphi\left(\frac{\left|x^{\prime \prime}\right|}{L}\right) B_{s+1} u\right\|+\left\|\left[\varphi\left(\frac{\left|x^{\prime \prime}\right|}{L}\right), P\right]\left(A_{s} u\right)\right\| \\
& \leq c\|P u\|_{H^{0,0, s}(\Omega)}+\left\|\varphi\left(\frac{\left|x^{\prime \prime}\right|}{L}\right) C_{s+1} u\right\|+\left\|\varphi\left(\frac{\left|x^{\prime \prime}\right|}{L}\right) D_{s} u\right\|+\varepsilon\|u\|_{H^{0,0, s}(\Omega)} \\
& \leq c\left(\|P u\|_{H^{0,0, s}(\Omega)}+\|u\|_{H^{0,0, s}(\Omega)}\right)+\varepsilon\|u\|_{H^{0,0, s}(\Omega)}+\left\|\varphi\left(\frac{\left|x^{\prime \prime}\right|}{L}\right) C_{s+1} u\right\| .
\end{aligned}
$$


We remember that $C_{s+1}$ is a pseudodifferential operator endowed with the symbol

$$
c\left(x, \xi^{\prime}\right)=-\frac{1}{i} \sum_{p=m+1}^{n}\left(x_{0}+\lambda-\alpha\left(x^{\prime}\right)\right)^{2} \sum_{i, j=m+1}^{n} \partial_{x_{p}} b_{j}\left(x^{\prime \prime}\right) \xi_{i}^{\prime \prime} \xi_{j}^{\prime \prime} \partial_{\xi_{p}}\left(1+\left|\xi^{\prime \prime}\right|^{2}\right)^{\frac{1}{2}} .
$$

Therefore, we have

$$
\begin{aligned}
C_{s+1} u= & \frac{1}{(2 \pi)^{n-m}} \int_{\mathbb{R}^{n-m}} e^{i x^{\prime \prime} \cdot \xi^{\prime \prime}} c\left(x, \xi^{\prime \prime}\right) \widehat{u}\left(x_{0}, x^{\prime}, \xi^{\prime \prime}\right) d \xi^{\prime \prime} \\
= & \frac{1}{(2 \pi)^{n-m}} \int_{\mathbb{R}^{n-m}} e^{i x^{\prime \prime} \cdot \xi^{\prime \prime}} \chi\left(\left|\xi^{\prime \prime}\right|\right) c\left(x, \xi^{\prime \prime}\right) \widehat{u}\left(x_{0}, x^{\prime}, \xi^{\prime \prime}\right) d \xi^{\prime \prime} \\
& +\frac{1}{(2 \pi)^{n-m}} \int_{\mathbb{R}^{n-m}} e^{i x^{\prime \prime} \cdot \xi^{\prime \prime}}\left(1-\chi\left(\left|\xi^{\prime \prime}\right|\right)\right) c\left(x, \xi^{\prime \prime}\right) \widehat{u}\left(x_{0}, x^{\prime}, \xi^{\prime \prime}\right) d \xi^{\prime \prime},
\end{aligned}
$$

where $\chi \in C_{0}^{\infty}(\mathbb{R})$ such that $\chi(t)=1$ for $|t|<1$. Therefore, we have

$$
\begin{aligned}
C_{s+1} u= & R u \\
& +\frac{1}{(2 \pi)^{n-m}} \int_{\mathbb{R}^{n-m}} e^{i x^{\prime \prime} \cdot \xi^{\prime \prime}}\left(1-\chi\left(\left|\xi^{\prime \prime}\right|\right)\right) c\left(x, \xi^{\prime \prime}\right) \widehat{u}\left(x_{0}, x^{\prime}, \xi^{\prime \prime}\right) d \xi^{\prime \prime},
\end{aligned}
$$

where $R$ is a regularizing operator. On the other hand, we have

$$
\begin{aligned}
& \int_{\mathbb{R}^{n-m}} e^{i x^{\prime \prime} \cdot \xi^{\prime \prime}}\left(1-\chi\left(\left|\xi^{\prime \prime}\right|\right)\right) c\left(x, \xi^{\prime \prime}\right) \widehat{u}\left(x_{0}, x^{\prime}, \xi^{\prime \prime}\right) d \xi^{\prime \prime} \\
& =\sum_{j=m+1}^{n} \int_{\mathbb{R}^{n-m}} e^{i x^{\prime \prime} \cdot \xi^{\prime \prime}} c^{\prime}\left(x, \xi^{\prime \prime}\right)\left(x_{0}+\lambda-\alpha\left(\widehat{\left.\left.x^{\prime}\right)\right)^{2} \partial_{x_{j}}} u\left(x_{0}, x^{\prime}, \xi^{\prime \prime}\right) d \xi^{\prime \prime},\right.\right.
\end{aligned}
$$

where $c^{\prime}\left(x, \xi^{\prime \prime}\right)=\frac{\left(1-\chi\left(\left|\xi^{\prime \prime}\right|\right)\right) c\left(x, \xi^{\prime \prime}\right)}{\left(x_{0}+\lambda-\alpha\left(x^{\prime}\right)\right)^{2}\left|\xi^{\prime \prime}\right|^{2}}\left(\xi_{1}+\cdots+\xi_{n}\right)$ is a symbol of order $s$. As a consequence, it follows that

$$
\begin{aligned}
C_{s+1} u= & R u+\sum_{j=1}^{n} C_{s}^{\prime}\left(x_{0}+\lambda-\alpha\left(x^{\prime}\right)\right)^{2} \partial_{x_{j}} u \\
= & R u+\sum_{j=m+1}^{n} C_{s}^{\prime}\left(x_{0}+\lambda-\alpha\left(x^{\prime}\right)\right)^{2} \partial_{x_{j}} \chi\left(\frac{\left|x_{0}+\lambda-\alpha\left(x^{\prime}\right)\right|}{\delta}\right) u \\
& +\sum_{j=m+1}^{n} C_{s}^{\prime}\left(x_{0}+\lambda-\alpha\left(x^{\prime}\right)\right)^{2} \partial_{x_{j}}\left[1-\chi\left(\frac{\left|x_{0}+\lambda-\alpha\left(x^{\prime}\right)\right|}{\delta}\right)\right] u .
\end{aligned}
$$

Then, taking into account Lemma 4.5,

$$
\begin{aligned}
\left\|C_{s+1} u\right\| \leq & \|R u\|+c \sum_{j=m+1}^{n}\left\|\frac{\left(x_{0}+\lambda-\alpha\left(x^{\prime}\right)\right)^{2}}{\delta} \delta \chi\left(\frac{\left|x_{0}+\lambda-\alpha\left(x^{\prime}\right)\right|}{\delta}\right) \partial_{x_{j}} u\right\|_{H^{0,0, s(\Omega)}} \\
& +c \sum_{j=m+1}^{n}\left\|\left(x_{0}+\lambda-\alpha\left(x^{\prime}\right)\right)^{2} \partial_{x_{j}}\left[1-\chi\left(\frac{\left|x_{0}+\lambda-\alpha\left(x^{\prime}\right)\right|}{\delta}\right)\right] u\right\|_{H^{0,0, s}(\Omega)}
\end{aligned}
$$




$$
\begin{aligned}
\leq & \|u\|_{H^{0,0, s}(\Omega)}+c \delta \sum_{j=m+1}^{n}\left\|\left(x_{0}+\lambda-\alpha\left(x^{\prime}\right)\right) \partial_{x_{j}} u\right\|_{H^{0,0, s}(\Omega)} \\
& +c \varepsilon\left\|P\left(1-\chi\left(\frac{\left|x_{0}+\lambda-\delta\left(x^{\prime}\right)\right|}{\delta}\right)\right) u\right\| \\
\leq & \|u\|_{H^{0,0, s}(\Omega)}+c \delta \sum_{j=m+1}^{n}\left\|\left(x_{0}+\lambda-\alpha\left(x^{\prime}\right)\right) \partial_{x_{j}} u\right\|_{H^{0,0, s}(\Omega)}+c \varepsilon\|P u\|_{H^{0,0, s}(\Omega)} \\
& +c \varepsilon\left\|\left[P,\left(1-\chi\left(\frac{\left|x_{0}+\lambda-\alpha\left(x^{\prime}\right)\right|}{\delta}\right)\right)\right](u)\right\|_{H^{0,0, s}(\Omega)} \\
\leq & \|u\|_{H^{0,0, s}(\Omega)}+c \delta \sum_{j=m+1}^{n}\left\|\left(x_{0}+\lambda-\alpha\left(x^{\prime}\right)\right) \partial_{x_{j}} u\right\|_{H^{0,0, s}(\Omega)} \\
& +c \varepsilon\left(\|P u\|_{H^{0,0, s}(\Omega)}+\left\|\partial_{x_{0}} u\right\|_{H^{0,0, s}(\Omega)}+\sum_{j=1}^{m}\left\|\partial_{x_{j}} u\right\|_{H^{0,0, s}(\Omega)}\right)
\end{aligned}
$$

Making use of (50), (51), and Lemma 3.1, we have

$$
\begin{aligned}
\left\|P v_{s}\right\| \leq & c\left(\|P u\|_{H^{0,0, s}\left(\Omega_{k}\right)}+k\left\|\partial_{x_{0}} u\right\|_{H^{0,0, s}\left(\Omega_{k}\right)}\right) \\
& +c \varepsilon\left(\|P u\|_{H^{0,0, s}\left(\Omega_{k}\right)}+\left\|\partial_{x_{0}} u\right\|_{H^{0,0, s}\left(\Omega_{k}\right)}+\sum_{j=1}^{m}\left\|\partial_{x_{j}} u\right\|_{H^{0,0, s}\left(\Omega_{k}\right)}\right) \\
& +c \delta \sum_{j=m+1}^{n}\left\|\left(x_{0}+\lambda-\alpha\left(x^{\prime}\right)\right) \partial_{x_{j}} u\right\|_{H^{0,0, s}\left(\Omega_{k}\right)} .
\end{aligned}
$$

Finally, by (52), (38), (39), (40), and (41), for $\delta$ and $\varepsilon$ small enough and, hence, $k$ small enough, as a result

$$
\begin{gathered}
\left\|\partial_{x_{0}} u\right\|_{H^{0,0, s\left(\Omega_{k}\right)}}+\sum_{j=1}^{m}\left\|\partial_{x_{j}} u\right\|_{H^{0,0, s}\left(\Omega_{k}\right)}+\sum_{j=m+1}^{n}\left\|\left(x_{0}+\lambda-\alpha\left(x^{\prime}\right)\right) \partial_{x_{j}} u\right\|_{H^{0,0, s}\left(\Omega_{k}\right)} \\
+\|u\|_{H^{0,0, s}\left(\Omega_{k}\right)} \leq c\|P u\|_{H^{0,0, s}\left(\Omega_{k}\right)}, \quad \forall u \in C_{0}^{\infty}(\bar{\Omega}): \operatorname{supp} u \subseteq \Omega_{k} .
\end{gathered}
$$

\section{A local existence theorem near the boundary and a regularity result}

Let $\Omega_{k}=\left[0, k\left[\times \Omega_{0}\right.\right.$, with $k>0$; the following local existence theorem near the boundary holds.

Theorem 5.1 Let $f \in H^{0,0, s}(\Omega)$, with $s \geq 0$. Then there exists $w \in H^{0,0, s}\left(\Omega_{k}\right)$ such that

$$
\left(w,{ }^{t} P u\right)=(f, u), \quad \forall u \in C_{0}^{\infty}(\bar{\Omega}): \operatorname{supp} u \subseteq \Omega_{k} .
$$

Proof Let $S$ be the space

$$
S=\left\{\psi \in C_{0}^{\infty}\left(\bar{\Omega}_{k}\right): \psi={ }^{t} P u, \forall u \in C_{0}^{\infty}(\bar{\Omega}): \operatorname{supp} u \subseteq \Omega_{k}\right\}
$$

Let $T$ be the linear functional defined as

$$
T(\psi)=T\left({ }^{t} P u\right)=(f, u), \quad \forall \psi \in S .
$$


Making use of Theorems 4.1 and 4.2, we have

$$
\begin{aligned}
|T(\psi)| & =|(f, u)| \\
& \leq\|f\|_{H^{0,0, s}(\Omega)}\|u\|_{H^{0,0,-s}\left(\Omega_{k}\right)} \\
& \leq c\|f\|_{H^{0,0, s}(\Omega)}\left\|{ }^{t} P u\right\|_{H^{0,0,-s}\left(\Omega_{k}\right)} \\
& =c^{\prime}\|\psi\|_{H^{0,0,-s}\left(\Omega_{k}\right)}, \quad \forall \psi \in S,
\end{aligned}
$$

where $c^{\prime}=c\|f\|_{H^{0,0, s}(\Omega)}$. Hence $T$ is continuous on $S$ and can be extended to a linear continuous functional in $H^{0,0,-s}\left(\bar{\Omega}_{k}\right)$. Making use of the representation theorems, there exists $w \in H^{0,0, s}\left(\bar{\Omega}_{k}\right)$ such that

$$
\begin{aligned}
T(\psi) & =(w, \phi) \\
& =\left(w,{ }^{t} P u\right) \\
& =(f, u), \quad \forall u \in C_{0}^{\infty}(\Omega): \operatorname{supp} u \subseteq \Omega_{k} .
\end{aligned}
$$

Now, let us study the regularity of the solution $w$. To this aim, we set

$$
L=D_{x_{0}}^{2}-\operatorname{Div}_{x^{\prime}}\left(A\left(x^{\prime}, x^{\prime \prime}\right) D_{x^{\prime}}\right)
$$

and, for every $x^{\prime \prime} \in \Omega^{\prime \prime}$, we consider the Cauchy problem

$$
\left\{\begin{array}{l}
L v=h, \quad \text { in }] 0, k\left[\times \Omega^{\prime},\right. \\
v\left(0, x^{\prime}\right)=0, \quad v_{x_{0}}\left(0, x^{\prime}\right)=0 .
\end{array}\right.
$$

Since $L$ is a strictly hyperbolic operator, it is well known that if $h \in H^{s}$ then the solution $v \in H^{s+1}$. As a consequence, since $P w=f$ in the sense of distributions, $L w=h$, with $h=$ $f+\left(x_{0}+\lambda-\alpha\left(x^{\prime}\right)\right)^{2} \operatorname{Div}_{x^{\prime \prime}}\left(B\left(x^{\prime \prime}\right) D_{x^{\prime \prime}}\right) w-\gamma(x) w$. Moreover, having $f \in H^{s, 2(r-s)}$, with $0 \leq s \leq r$ and $r \geq 2$, it follows that

$$
w \in H^{1,2(r-1)}\left(\Omega_{k}\right) .
$$

Let us proceed by induction. We prove

$$
w \in H^{s-1,2(r-1)}\left(\Omega_{k}\right) \quad \Longrightarrow \quad w \in H^{s, 2(r-s)}\left(\Omega_{k}\right), \quad 2 \leq s \leq r .
$$

Hence, we compute

$$
\partial^{s-1,2(r-s+1)} L w=\partial^{s-1,2(r-s+1)} h,
$$

from which we have

$$
\begin{aligned}
L \partial^{s-1,2(r-s+1)} w & =\partial^{s-1,2(r-s+1)} h-\left[\partial^{s-1,2(r-s+1)}, L\right] w \\
& =\partial^{s-1,2(r-s+1)} f+\left(x_{0}+\lambda-\alpha\left(x^{\prime}\right)\right)^{2} \operatorname{Div}_{x^{\prime \prime}}\left(B\left(x^{\prime \prime}\right) D_{x^{\prime \prime}}\right) \partial^{s-1,2(r-s+1)} w
\end{aligned}
$$




$$
\begin{aligned}
& +\left[\left(x_{0}+\lambda-\alpha\left(x^{\prime}\right)\right)^{2} \operatorname{Div}_{x^{\prime \prime}}\left(B\left(x^{\prime \prime}\right) D_{x^{\prime \prime}}\right), \partial^{s-1,2(r-s+1)}\right] w \\
& -\left[\partial^{s-1,2(r-s+1)}, L\right] w .
\end{aligned}
$$

This implies

$$
w \in H^{s, 2(r-s+1)}\left(\Omega_{k}\right) \subseteq H^{s, 2(r-s)}\left(\Omega_{k}\right) .
$$

Since $s \leq r-1$, as a result

$$
w \in H^{r}\left(\Omega_{k}\right)
$$

Therefore, we proved that if $w$ is solution to the equation:

$$
\left(w,{ }^{t} P \varphi\right)=(f, \varphi), \quad \forall \varphi \in C_{0}^{\infty}\left(\bar{\Omega}_{k}\right),
$$

then the distribution $w \in H^{r+1}\left(\bar{\Omega}_{k}\right)(r \geq 2)$. Integrating by part the left-hand side of (54), as a result, for every $\varphi \in C_{0}^{\infty}\left(\bar{\Omega}_{k}\right)$ with $\operatorname{supp} \varphi \subseteq \Omega_{k}$,

$$
(P w, \varphi)=(f, \varphi),
$$

and that implies

$$
P w=f, \quad \text { a.e. in } \Omega_{k} \text {. }
$$

Moreover, integrating by parts the left-hand side of (54), for every $\varphi \in C_{0}^{\infty}\left(\bar{\Omega}_{k}\right)$ with $\varphi\left(0, x^{\prime}, x^{\prime \prime}\right)=0$, we have

$$
(P w, \varphi)-\int_{\Omega_{0}} w\left(0, x^{\prime}, x^{\prime \prime}\right) \varphi_{x_{0}}\left(0, x^{\prime}, x^{\prime \prime}\right) d x^{\prime} d x^{\prime \prime}=(f, \varphi),
$$

and combining with (55), it follows that

$$
w\left(0, x^{\prime}, x^{\prime \prime}\right)=0 .
$$

Finally, integrating by part the left-hand side of (54), for every $\varphi \in C_{0}^{\infty}\left(\Omega_{k}\right)$ with $\varphi_{x_{0}}\left(0, x^{\prime}\right.$, $\left.x^{\prime \prime}\right)=0$, we obtain

$$
(P w, \varphi)-\int_{\Omega_{0}} w_{x_{0}}\left(0, x^{\prime}, x^{\prime \prime}\right) \varphi\left(0, x^{\prime}, x^{\prime \prime}\right) d x^{\prime} d x^{\prime \prime}=(f, \varphi),
$$

and making use of (55), as a result

$$
w_{x_{0}}\left(0, x^{\prime}, x^{\prime \prime}\right)=0 \text {. }
$$

Hence, we proved that $w \in H^{r}\left(\Omega_{k}\right)(r \geq 2)$ is a solution to the problem

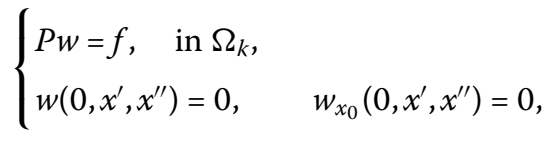

for $k$ small enough. 


\section{A global existence result}

Let $\bar{x}_{0}>0$ and let $\Omega_{\bar{x}_{0}}=\left[\bar{x}_{0},+\infty\left[\times \Omega_{0}\right.\right.$, by means of the change of variables $x_{0}=y_{0}+\bar{x}_{0}$, the problem

$$
\left\{\begin{array}{l}
P w=f, \quad \text { in } \Omega_{\bar{x}_{0}}, \\
w\left(\bar{x}_{0}, x^{\prime}, x^{\prime \prime}\right)=0, \quad w_{x_{0}}\left(\bar{x}_{0}, x^{\prime}, x^{\prime \prime}\right)=0,
\end{array}\right.
$$

becomes

$$
\left\{\begin{array}{l}
P v=f, \quad \text { in } \Omega, \\
v\left(0, x^{\prime}, x^{\prime \prime}\right)=0, \quad v_{y_{0}}\left(0, x^{\prime}, x^{\prime \prime}\right)=0
\end{array}\right.
$$

where $v\left(y_{0}, x^{\prime}, x^{\prime \prime}\right)=v\left(x_{0}-\bar{x}_{0}, x^{\prime}, x^{\prime \prime}\right)=w\left(x_{0}, x^{\prime}, x^{\prime \prime}\right)$.

According to the results of Section 5 , for $k$ small enough, there exists a solution $v \in$ $H^{r}\left(\Omega_{k}\right), r \geq 2$, verifying the problem

$$
\left\{\begin{array}{l}
P v=f, \quad \text { in } \Omega_{k}, \\
v\left(0, x^{\prime}, x^{\prime \prime}\right)=0, \quad v_{y_{0}}\left(0, x^{\prime}, x^{\prime \prime}\right)=0 .
\end{array}\right.
$$

Hence, there exists a solution $w \in H^{r}\left(\Omega_{\bar{x}_{0}, k}\right)$, where $\Omega_{\bar{x}_{0}, k}=\left[\bar{x}_{0}, \bar{x}_{0}+k\left[\times \Omega_{0}\right.\right.$ verifying the problem

$$
\left\{\begin{array}{l}
P w=f, \quad \text { in } \Omega_{\bar{x}_{0}, k}, \\
w\left(\bar{x}_{0}, x^{\prime}, x^{\prime \prime}\right)=0, \quad w_{x_{0}}\left(\bar{x}_{0}, x^{\prime}, x^{\prime \prime}\right)=0 .
\end{array}\right.
$$

Now, if $B\left(x^{\prime \prime}\right)$ is constant and $\Omega_{0}=\Omega^{\prime} \times \mathbb{R}^{n-m}, k$ does not depend on $s$. Then we can proceed in the following way. From the existence of a solution $w \in H^{r}\left(\Omega_{\bar{x}_{0}, k}\right)$ to problem (56), it follows that also the problem

$$
\left\{\begin{array}{l}
P w=f, \quad \text { in } \Omega_{\bar{x}_{0}, k} \\
w\left(\bar{x}_{0}, x^{\prime}, x^{\prime \prime}\right)=g_{1}\left(x^{\prime}, x^{\prime \prime}\right), \quad w_{x_{0}}\left(\bar{x}_{0}, x^{\prime}, x^{\prime \prime}\right)=g_{2}\left(x^{\prime}, x^{\prime \prime}\right)
\end{array}\right.
$$

where $f \in C^{\infty}(\Omega), g_{1} \in C^{\infty}\left(\Omega_{0}\right), g_{2} \in C^{\infty}\left(\Omega_{0}\right)$, admits a solution $w \in C^{\infty}\left(\bar{\Omega}_{\bar{x}_{0}, k}\right)$. In fact, let $h\left(x_{0}, x^{\prime}, x^{\prime \prime}\right)$ be a function belonging to $C^{\infty}\left(\Omega_{\bar{x}_{0}, k}\right)$ such that $h\left(\bar{x}_{0}, x^{\prime}, x^{\prime \prime}\right)=g_{1}\left(x^{\prime}, x^{\prime \prime}\right)$ and $h_{x_{0}}\left(\bar{x}_{0}, x^{\prime}, x^{\prime \prime}\right)=g_{2}\left(x^{\prime}, x^{\prime \prime}\right)$, the solution to (57) is $w=h+\bar{w}$, where $\bar{w}$ is solution to

$$
\left\{\begin{array}{l}
P \bar{w}=f+P h, \quad \text { in } \Omega_{\bar{x}_{0}, k}, \\
\bar{w}\left(\bar{x}_{0}, x^{\prime}, x^{\prime \prime}\right)=0, \quad \bar{w}_{x_{0}}\left(\bar{x}_{0}, x^{\prime}, x^{\prime \prime}\right)=0 .
\end{array}\right.
$$

Set $\Omega_{h}=\left[0, h\left[\times \Omega_{0}\right.\right.$, with $h>0$, by means of compactness theorems and the arbitrariness of $\bar{x}_{0}$, we can decompose $\bar{\Omega}_{k}$ in the union of a finite number of compacts $\bar{\Omega}_{i}=\left[k_{i-1}, k_{i}\right] \times$ $\Omega_{0}$, for $i=1, \ldots, p$, where $k_{0}=0$, and such that there exists a solution $w_{i} \in C^{\infty}\left(\Omega_{i}\right)$ to the problem

$$
\left\{\begin{array}{l}
P w_{i}=f, \quad \text { in } \Omega_{i}, \\
w_{i}\left(k_{i}, x^{\prime}, x^{\prime \prime}\right)=w_{i-1}\left(k_{i}, x^{\prime}, x^{\prime \prime}\right), \quad \partial_{x_{0}} w_{i}\left(k_{i}, x^{\prime}, x^{\prime \prime}\right)=\partial_{x_{0}} w_{i-1}\left(k_{i}, x^{\prime}, x^{\prime \prime}\right),
\end{array}\right.
$$


where $i=1, \ldots, p-1, w_{0}\left(0, x^{\prime}, x^{\prime \prime}\right)=0$ and $\partial_{x_{0}} w_{0}\left(0, x^{\prime}, x^{\prime \prime}\right)=0$. By construction, it follows that the function

$$
w\left(x_{0}, x^{\prime}, x^{\prime \prime}\right)=\sum_{i=0}^{p} w_{i}\left(x_{0}, x^{\prime}, x^{\prime \prime}\right) \chi_{i}\left(x_{0}, x^{\prime}, x^{\prime \prime}\right),
$$

where

$$
\chi_{i}\left(x_{0}, x^{\prime}, x^{\prime \prime}\right)= \begin{cases}1 & \text { in }\left[k_{i}, k_{i+1}\right] \times \Omega_{0} \\ 0 & \text { otherwise }\end{cases}
$$

is a solution to the problem

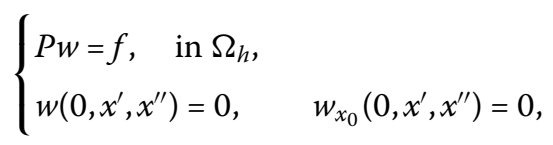

with $f \in C^{\infty}(\bar{\Omega})$ and $w \in C^{\infty}\left(\Omega_{h}\right)$. For the arbitrariness of $h$, we have proved that under assumptions (i), (ii), (iii), and (iv), if $\Omega_{0}=\Omega^{\prime} \times \mathbb{R}^{n-m}$ and $B\left(x^{\prime \prime}\right)$ is a constant, then the problem

$$
\left\{\begin{array}{l}
P u=f, \quad \text { in } \Omega, \\
u\left(0, x^{\prime}, x^{\prime \prime}\right)=0, \quad u_{x_{0}}\left(0, x^{\prime}, x^{\prime \prime}\right)=0,
\end{array}\right.
$$

with $f \in C^{\infty}(\bar{\Omega})$, admits a solution $u \in C^{\infty}(\bar{\Omega})$.

If $B\left(x^{\prime \prime}\right)$ is not constant, since $c$ depends on $s$ in (37), we proceed as follows. For every $h>0$ and for every $\bar{x}_{0} \in\left[0, h\left[\right.\right.$, we set $\Omega_{\bar{x}_{0}, k}=\left[\bar{x}_{0}, \bar{x}_{0}+k\left[\times \Omega_{0}\right.\right.$. By means of a change of variables $x_{0}=y_{0}+\bar{x}_{0}$, we show, as done before, (37) for every $u \in C_{0}^{\infty}\left(\bar{\Omega}_{\bar{x}_{0}, k}\right)$ and $k$ small enough. Then it is possible to divide $\Omega_{h}$ in a finite number of subsets $\Omega_{0}=\left[0, k_{0}\left[\times \Omega_{0}\right.\right.$, $\Omega_{1}=\left[k_{1}, k_{2}\left[\times \Omega_{0}, \ldots, \Omega_{p}=\left[k_{p}, h\left[\times \Omega_{0}\right.\right.\right.\right.$, with $k_{i+1}<k_{i}<k_{j}$, for every $i=0, \ldots, p, k_{p+1}=h$ and $j \geq i+2$, such that (48) holds in every $\Omega_{i}$, namely (37) holds for every $u \in C_{0}^{\infty}\left(\bar{\Omega}_{i}\right)$, $i=0, \ldots, p$. Now, for every $u \in C^{\infty}(\bar{\Omega})$ with supp $u \subseteq \Omega_{h}$, as a result

$$
\begin{aligned}
\|u\|_{H^{0,0, s}\left(\left[k_{i}, k_{i+2}\left[\times \Omega_{0}\right)\right.\right.} \leq & \|u \psi\|_{H^{0,0, s}\left(\left[k_{i}, k_{i+1}\left[\times \Omega_{0}\right)\right.\right.} \\
\leq & c\|P u \psi\|_{H^{0,0, s}\left(\left[k_{i}, k_{i+1}\left[\times \Omega_{0}\right)\right.\right.} \\
\leq & c\|P u\|_{H^{0,0, s}\left(\Omega_{h}\right)} \\
& +c\left(\left\|\partial_{x_{0}} u\right\|_{H^{0,0, s}\left[\left[k_{i+2}, k_{i+4}\left[\times \Omega_{0}\right)\right.\right.}+\|u\|_{H^{0,0, s}\left(\left[k_{i+2}, k_{i+4}\left[\times \Omega_{0}\right)\right.\right.}\right),
\end{aligned}
$$

where $\psi \in C^{\infty}\left(\left[0, h[), \psi=1\right.\right.$ on $\left[k_{i}, k_{i+2}\right]$ and supp $\psi \subseteq\left[k_{i}, k_{i+1}\right]$, for every $i$ odd with $i=$ $-1, \ldots, p-2, k_{-1}=0$ and $k_{p+2}>k_{p+1}$. By (58) it follows that

$$
\|u\|_{H^{0,0, s}\left(\left[k_{i}, k_{i+2}\left[\times \Omega_{0}\right)\right.\right.} \leq c\|P u\|_{H^{0,0, s}\left(\Omega_{h}\right)}
$$

from which, adding with respect to $i$, with $i$ odd and $i=-1, \ldots, p-2$, as a result

$$
\|u\|_{H^{0,0, s}\left(\Omega_{h}\right)} \leq c\|P u\|_{H^{0,0, s}\left(\Omega_{h}\right)} .
$$


Making using of the previous inequality and proceeding as in Section 5, we see that there exists $w \in H^{0,0, s}\left(\Omega_{h}\right)$ such that

$$
\left(w,{ }^{t} P u\right)=(f, u), \quad \forall u \in C_{0}^{\infty}\left(\bar{\Omega}_{h}\right),
$$

and $w \in H^{r}\left(\Omega_{h}\right) \cap H^{r, 2(r-s)}\left(\Omega_{h}\right)$, with $0 \leq s \leq r, r \geq 2$. Integrating by parts in (59) (see Section 5), for the arbitrariness of $h$, we can prove that for every $h>0$ the problem

$$
\left\{\begin{array}{l}
P w=f, \quad \text { in } \Omega_{h}, \\
w\left(0, x^{\prime}, x^{\prime \prime}\right)=0, \quad w_{x_{0}}\left(0, x^{\prime}, x^{\prime \prime}\right)=0,
\end{array}\right.
$$

with $f \in H^{s, 2(r-s)}(\Omega)$, for every $0 \leq s \leq r, r \leq 2$, admits a solution $w \in H^{r}\left(\Omega_{h}\right) \cap H^{r, 2(r-s)}\left(\Omega_{h}\right)$, with $0 \leq s \leq r, r \geq 2$.

\section{Competing interests}

The authors declare that there is no conflict of interests regarding the publication of this paper.

\section{Authors' contributions}

All authors contributed equally in this article. They read and approved the final manuscript.

\section{Acknowledgements}

The first author was partially supported by STAR 2014 'Variational Analysis and Equilibrium Models in Physical and Socio-Economic Phenomena' (Grant 14-CSP3-C03-099). The authors cordially thank the referees for their valuable comments and suggestions, which lead to a clearer presentation of this work.

\section{Received: 14 April 2016 Accepted: 21 July 2016 Published online: 15 August 2016}

\section{References}

1. Hörmander, L: The Cauchy problem for differential equations with double characteristics. J. Anal. Math. 32, 118-196 (1977)

2. Bernardi, E, Parenti, C, Parmeggiani, A: The Cauchy problem for hyperbolic operators with double characteristics in presence of transition. Commun. Partial Differ. Equ. 37, 1315-1356 (2012)

3. Bernardi, E, Bove, A, Parenti, C: Geometric results for a class of hyperbolic operators with double characteristics. J. Funct. Anal. 116, 62-82 (1993)

4. Ivrii, VJ, Petkov, VM: Necessary conditions for well-posedness of the Cauchy problem for non-strictly hyperbolic equations. Russ. Math. Surv. 29, 1-70 (1974)

5. Iwasaki, N: The Cauchy problem for effectively hyperbolic equations (a special case). J. Math. Kyoto Univ. 23, 503-562 (1983)

6. Melrose, RB: The Cauchy problem for effectively hyperbolic operators. Hokkaido Math. J. 12, 371-391 (1983)

7. Melrose, RB: The Cauchy problem and propagations of singularities. In: Chen, SS (ed.) Seminar on Nonlinear Partial Differential Equations, vol. 2, pp. 185-201. Springer, Berlin (1984)

8. Nishitani, T: The effectively hyperbolic Cauchy problem. Lect. Notes Math. 1505, 71-167 (1991)

9. Nishitani, T: Local energy integral for effectively hyperbolic operators. J. Math. Kyoto Univ. 24, 623-658, 659-666 (1984)

10. Nishitani, T: Local and microlocal Cauchy problem for non-effectively hyperbolic operators. J. Hyperbolic Differ. Equ. $11,185-213(2014)$

11. Barbagallo, A, Esposito, V: A priori estimate for a class of hyperbolic equations with double characteristics. Rend. Accad. Sci. Fis. Mat. LXXXI, 113-120 (2014)

12. Barbagallo, A, Esposito, V: A global existence and uniqueness result for a class of hyperbolic operators. Ric. Mat. 63 , 25-40 (2014)

13. Barbagallo, A, Esposito, V: New results on the Cauchy problem for a class of hyperbolic equations in the half-space. AIP Conf. Proc. 1648, 1-4 (2015)

14. Barbagallo, A, Esposito, V: The Cauchy-Dirichlet problem for a class of hyperbolic operators with double characteristics in the presence of transition. J. Math. Anal. Appl. 442, 243-249 (2016)

15. Barbagallo, A, Esposito, V: Energy estimates for the Cauchy problem associated to a class of hyperbolic operators with double characteristics in presence of transition. Ric. Mat. 64, 243-249 (2015)

16. Treves, F: Introduction to Pseudodifferential and Fourier Integral Operators, vol. 1. Springer, Berlin (1980) 